\title{
An on-line time dependent parametric model order reduction scheme with focus on dynamic stress recovery
}

\author{
T. Tamarozzia ${ }^{\mathrm{a}, *}$, G.H.K. Heirman ${ }^{\mathrm{a}}$, W. Desmet ${ }^{\mathrm{a}}$ \\ ${ }^{a}$ Department of Mechanical Engineering, KU Leuven, Celestijnenlaan 300B, 3001 Leuven, Belgium
}

\begin{abstract}
In view of the tendency towards ever lighter and more powerful machines and even shorter design cycles, it becomes essential to have virtual prototyping tools that allow for fast and reliable numerical simulations. Current state-of-the-art structural dynamics and flexible multibody simulation techniques usually involve the solution of matrix systems with thousands to millions of variables. Model order reduction schemes are used to keep computational effort affordable at the expense of a minimal loss of accuracy. These techniques typically face difficulties with systems in which flexible bodies can be loaded in many degrees of freedom and rarely allow for accurate local stress and strain evaluation. The present work proposes to address both issues for the particular but frequent case of moving loads or boundary conditions. This behavior is found in most of the contact problems and systems that include sliding components for which many loading or boundary locations are possible but only a few of them are active at a certain moment in time. The proposed scheme exploits a reduction vector space that continuously varies in time by means of a parametric definition of the external load position. Contrary to the majority of the parametric model order reduction schemes that allow mainly for quasi-static parametric variations, the proposed approach can be used efficiently for time simulation of dynamically varying parametric models. This is achieved by considering the implicit time dependency of the reduction vector space using Galerkin projections or alternatively by direct substitution of the reduced kinetic energy, potential energy and generalized forces in the Lagrange equations. It is shown, by developing a consistent mathematical framework, that the price to pay for the very compact reduction space obtained is the evaluation of some extra terms in the equations of motion. Numerical examples are used to assess the accuracy of the proposed method. Results show the potential of this strategy with particular focus on displacement and stress fields and furthermore highlight its real-time potential. Moreover the developed framework together with the numerical results allow for a deeper physical understanding of the complex phenomena related to this category of time varying multiple-input/multiple output systems.
\end{abstract}

Keywords: parametric model order reduction, stress recovery, flexible bodies, static augmentation, time varying load location

${ }^{*}$ Corresponding Author: Tommaso Tamarozzi, Dept. Mechanical Engineering, KU Leuven, Celestijnenlaan 300B, 3001 Leuven, Belgium, Tel.: +32 16 328608, Fax +32 16322987

Email address: tommaso.tamarozzi@mech.kuleuven. be (T. Tamarozzi) 


\section{Introduction and state of the art}

Current state-of-the-art structural dynamics and flexible multibody simulation (FMBS) techniques usually involve the solution of systems with thousands to millions of variables especially when the Finite Element (FE) method is used for the spatial discretization of the system.

Model order reduction (MOR) schemes are used to reduce the size of the problem keeping computational effort affordable with a minimal loss of accuracy. These techniques typically face difficulties with systems in which flexible bodies can be loaded in many degrees of freedom [1,2]. This is due to the fact that a large set of input/output (I/O) interfaces, automatically translates into a large set of so called correction shape vectors to be included in the reduction space used to decrease the model size. The majority of MOR techniques suffer from this strong I/O correlation. As an example one or more new shape vectors need to be included in the reduction space for each $\mathrm{I} / \mathrm{O}$ point if static completeness and good dynamic approximation need to be preserved [3]. This is especially true when stresses and strains are of interest.

The present work addresses both issues for the particular but frequent case of moving loads or boundary conditions. This behavior is found in most of the contact problems and systems that include sliding components for which many loading or boundary locations are possible but only a few of them are active at a certain moment in time. With the typical example of a pair of meshing gears in mind, it becomes apparent that all the nodes of a gear FE mesh on the flanks of all teeth are possible contact locations during a full revolution. It is clear though, that only a few of those nodes will be loaded at a certain moment in time, i.e. usually a small number of nodes on the flanks of a limited set of teeth. The problem related to this time varying multipleinput/multiple-output (MIMO) behavior was previously addressed by using a discontinuously changing reduction space as in static modes switching (SMS) [4]. This was developed in a FMBS framework with focus on gear contact [5]. In [4,6] it is shown that this technique may lead to numerical difficulties due to its non-smooth characteristics. This calls for the use of numerical schemes with desirable damping properties or physical damping in the system and makes the approach more challenging for less experienced users. Another approach found in literature, proposes a quasi-static consideration of the correction shape vectors by solving a large linear system of equations instead of a smaller but numerically costly matrix inversion [7]. The latter approach is applicable to ordinary differential equations (ODE) while its use in FMBS, where typically differential algebraic equations (DAE) need to be solved is not a straight-forward task.

The scheme proposed in this work is developed to retain as much as possible the simplicity of the SMS technique while at the same time eliminating the numerical difficulties inherent with the method. This would allow its use to less experienced users and for a broader range of problems. Particular attention is given to the issue of accurate dynamic stress recovery. With the goal of clearly presenting the difficulties related to stress recovery using model order reduction techniques and to introduce the concept of parametric model order reduction (PMOR), a brief review of these techniques is presented first.

\subsection{MOR and Parametric MOR techniques}

In structural dynamics or FMBS, when using the FE method, the number of degrees of freedom (DOF) is usually very large so that the deformation of each body is approximated by using a reduced set of shape vectors instead of the complete set of nodal coordinates. The choice of the vector set used to represent body flexibility frequently distinguishes one MOR technique from another. A vast literature on the topic is available, see e.g. [3, 8-10]. For the present work, that focuses on systems with variable MIMO characteristics, it is necessary for a subset of the 
shape vectors, to have a one-to-one relation with the spatial location of the loads or boundary conditions. Anticipating what is more thoroughly explained in section 2, the wording 'one-toone relation' is used to define a shape vector that is obtained by statically applying a unit load on a certain DOF. Furthermore any operation applied to it, e.g. projections, orthonormalizations and rotations, should not brake the link to that particular DOF. As an example, each shape vector belonging to a set of static deformations relates to the specific DOF to which the load is applied but this property does not hold anymore if a mass-orthogonalization procedure is performed. By adopting the step-by-step pre-processing procedure defined in $[4,5]$ the desired one-to-one relation can be obtained starting from any vector set. The proposed approach, though, is readily applicable if, e.g. the modal technique with static augmentation, modal technique with frequency response modes (FRM) or the more recent Krylov based reductions are used. All these techniques have the advantage of fulfilling the dynamic boundary conditions and are potentially capable of exactly reproducing the static deformation or the steady-state behavior at discrete frequencies of the underlying FE model. The main drawback lies in the fact that one or more shape vectors have to be added to the reduction vector set for each $\mathrm{I} / \mathrm{O}$ location, often leading to large reduced order models (ROM) and potentially jeopardizing the success of the MOR strategy.

Consider, without loss of generality with respect to a damped system, the equations of motion for an undamped dynamical system in second-order form

$$
M \ddot{x}+K \boldsymbol{x}=\boldsymbol{B u}
$$

where $\boldsymbol{M}, \boldsymbol{K}, \boldsymbol{B}$, are respectively the mass, stiffness and input matrices, $\boldsymbol{x}$ is the vector of nodal displacements and $\boldsymbol{u}$ is the vector of inputs. The number of DOFs in the system can be consistently reduced by using a Galerkin projection. The system in eq. 1 can be projected on a vector space normal to its residual and spanned by the columns of a full-column rank matrix $\boldsymbol{V}$ containing a set of shape vectors as follows:

$$
\boldsymbol{V}^{T} \boldsymbol{M V} \ddot{\boldsymbol{x}}_{\boldsymbol{r}}+\boldsymbol{V}^{T} \boldsymbol{K} \boldsymbol{V} \boldsymbol{x}_{\boldsymbol{r}}=\boldsymbol{V}^{T} \boldsymbol{B} \boldsymbol{u}=\boldsymbol{M}_{\boldsymbol{r}} \ddot{\boldsymbol{x}}_{\boldsymbol{r}}+\boldsymbol{K}_{\boldsymbol{r}} \boldsymbol{x}_{\boldsymbol{r}}=\boldsymbol{B}_{\boldsymbol{r}} \boldsymbol{u}
$$

where $\boldsymbol{M}_{\boldsymbol{r}}, \boldsymbol{K}_{\boldsymbol{r}}, \boldsymbol{B}_{\boldsymbol{r}}$, are respectively the reduced mass, stiffness and input matrix and $\boldsymbol{x}_{\boldsymbol{r}}$ is the vector of reduced generalized coordinates with $\boldsymbol{x} \cong \tilde{\boldsymbol{x}}=\boldsymbol{V} \boldsymbol{x}_{\boldsymbol{r}}$.

In recent years, the field of parametric model order reduction has emerged [11-13]. The goal of this research branch is to extend MOR strategies to problems in which the system under analysis is dependent on one or more parameters. As an example the engineer might need to analyze the behavior of a system in which parameters defining material properties, geometry, boundary conditions or external load location can vary. A parameterized, undamped secondorder dynamical system can be generally represented as follows:

$$
M(p) \ddot{x}+K(p) x=B(p) u
$$

where $\boldsymbol{p}$ is a vector of parameters defining a certain system configuration. A successful PMOR technique should allow to find a particular optimal realization of the system without the need of solving eq. 3 for a large amount of realizations of $\boldsymbol{p}$. This goal can be achieved if the PMOR scheme is capable to retain the complex parameter dependency of the original problem, without the need of performing a new reduction step at each realization $\boldsymbol{p}$.

Several methods exist in literature. Without claim of completeness but aiming keep the discussion self-contained as much as possible, they are briefly revised in the following. 
One first category of parametric dependency allows to write the system matrices in eq. 3 as follows:

$$
\begin{gathered}
\boldsymbol{M}(\boldsymbol{p})=\boldsymbol{M}_{\mathbf{0}}+\sum_{n=1}^{N} f_{n}(\boldsymbol{p}) \boldsymbol{M}_{\boldsymbol{n}} \\
\boldsymbol{K}(\boldsymbol{p})=\boldsymbol{K}_{\mathbf{0}}+\sum_{n=1}^{N} g_{n}(\boldsymbol{p}) \boldsymbol{K}_{\boldsymbol{n}} \\
\boldsymbol{B}(\boldsymbol{p})=\boldsymbol{B}_{\mathbf{0}}+\sum_{n=1}^{N} h_{n}(\boldsymbol{p}) \boldsymbol{B}_{\boldsymbol{n}}
\end{gathered}
$$

in which $f_{n}, g_{n}$ and $h_{n}$ are linear or non-linear functions of the parameters. In this expression it is assumed that the dependency of the high fidelity model (HFM) on the parameters is affine or separable $[14,15]$. This is the case e.g. if the PMOR keeps its validity in a small perturbation region around a given parameter realization allowing for a meaningful Taylor expansion or if the parameter dependency can be expressed exactly by simple analytical expressions. Ideally, PMOR for these types of problems would result in the following reduced matrices that retain the parameter dependency explicitly:

$$
\begin{gathered}
\boldsymbol{M}_{\boldsymbol{r}}(\boldsymbol{p})=\boldsymbol{V}^{T} \boldsymbol{M}_{\mathbf{0}} \boldsymbol{V}+\boldsymbol{V}^{T}\left(\sum_{n=1}^{N} f_{n}(\boldsymbol{p}) \boldsymbol{M}_{\boldsymbol{n}}\right) \boldsymbol{V} ; \\
\boldsymbol{K}_{\boldsymbol{r}}(\boldsymbol{p})=\boldsymbol{V}^{T} \boldsymbol{K}_{\mathbf{0}} \boldsymbol{V}+\boldsymbol{V}^{T}\left(\sum_{n=1}^{N} g_{n}(\boldsymbol{p}) \boldsymbol{K}_{\boldsymbol{n}}\right) \boldsymbol{V} ; \\
\boldsymbol{B}_{\boldsymbol{r}}(\boldsymbol{p})=\boldsymbol{V}^{T} \boldsymbol{B}_{\mathbf{0}}+\boldsymbol{V}^{T}\left(\sum_{n=1}^{N} h_{n}(\boldsymbol{p}) \boldsymbol{B}_{\boldsymbol{n}}\right)
\end{gathered}
$$

This formulation allows for calculation of the parametric model in a pure pre-processing step. A milestone work on this topic was presented in [11]. The importance of including shape vectors that are capable of representing exactly the static deformation, is highlighted in the work. Several authors analyzed the particular case of eq.5, see $[11,12,16]$, e.g. using the concept of moment matching (e.g. Krylov based). In [16] the problem is addressed with particular focus on special single-input/single-output (SISO) parameterization and desirable characteristics of the proposed PMOR strategy for sensitivity analysis. As noted in [15], the category of problems in eq.4, even if highly relevant, represents only a limited subset of problems. Typically applicable solution methods suffer from the curse of dimensionality. When a large number of parameters, expansion points, moments to be matched (for Krylov based techniques) or snapshots (for proper orthogonal decomposition (POD) or balanced truncation (BT)) are necessary, the amount of shape vectors in the matrix $\boldsymbol{V}$ can become prohibitive. This might limit the success of the reduction strategy. As a partial solution to this problem, as proposed in [17], singular value decomposition (SVD) can be used to reduce further the size of the large models, slightly sacrificing accuracy.

A more general category of parametric dependency, that includes the subcases previously 
discussed, can be represented by writing the reduced system matrices in eq.2:

$$
\begin{gathered}
\boldsymbol{M}_{\boldsymbol{r}, \boldsymbol{i}}=\boldsymbol{V}_{\boldsymbol{i}}^{T}\left(\boldsymbol{p}_{\boldsymbol{i}}\right) \boldsymbol{M}\left(\boldsymbol{p}_{\boldsymbol{i}}\right) \boldsymbol{V}_{\boldsymbol{i}}\left(\boldsymbol{p}_{\boldsymbol{i}}\right) ; \boldsymbol{K}_{\boldsymbol{r}, \boldsymbol{i}}=\boldsymbol{V}_{\boldsymbol{i}}^{T}\left(\boldsymbol{p}_{\boldsymbol{i}}\right) \boldsymbol{K}\left(\boldsymbol{p}_{\boldsymbol{i}}\right) \boldsymbol{V}_{\boldsymbol{i}}\left(\boldsymbol{p}_{\boldsymbol{i}}\right) ; \boldsymbol{B}_{\boldsymbol{r}, \boldsymbol{i}}\left(\boldsymbol{p}_{\boldsymbol{i}}\right)=\boldsymbol{V}_{\boldsymbol{i}}^{T}\left(\boldsymbol{p}_{\boldsymbol{i}}\right) \boldsymbol{B}\left(\boldsymbol{p}_{\boldsymbol{i}}\right) \\
\boldsymbol{M}_{\boldsymbol{r}}(\boldsymbol{p})=\sum_{i=1}^{l} s_{i}(\boldsymbol{p}) \boldsymbol{M}_{\boldsymbol{r}, \boldsymbol{i}} \quad ; \quad \boldsymbol{K}_{\boldsymbol{r}}(\boldsymbol{p})=\sum_{i=1}^{l} s_{i}(\boldsymbol{p}) \boldsymbol{K}_{r, i} \quad ; \quad \boldsymbol{B}_{\boldsymbol{r}}(\boldsymbol{p})=\sum_{i=1}^{l} s_{i}(\boldsymbol{p}) \boldsymbol{B}_{\boldsymbol{r}, \boldsymbol{i}} \quad ;
\end{gathered}
$$

The subscript $i$ represents a specific parameter realization and so a specific reduced model. The matrix $\boldsymbol{V}\left(\boldsymbol{p}_{\boldsymbol{i}}\right)$ retains the parametric dependency and the system matrices $\boldsymbol{M}_{\boldsymbol{r}, \boldsymbol{i}}, \boldsymbol{K}_{\boldsymbol{r}, \boldsymbol{i}}, \boldsymbol{B}_{\boldsymbol{r}, \boldsymbol{i}}$ can be directly interpolated by means of a linear or non-linear function $s_{i}$. This approach allows, potentially, to interpolate reduced models computed at particular realizations of $\boldsymbol{p}$ directly. These local models can be optimally computed, in a certain defined sense, for each value of the sampled realizations $\boldsymbol{p}_{\boldsymbol{i}}$. In this case all the relevant matrices can be obtained in a pre-processing reduction step without on-line evaluation of the system at $\boldsymbol{p}$ realizations different from the sampled values.

The direct interpolation expressed by eq. 7 is not necessarily meaningful. As explained in $[14,15]$ the local projection matrices $\boldsymbol{V}_{\boldsymbol{i}}$ do not generally have a consistent physical interpretation. Specifically a direct interpolation of ROM discards the dependence of the generalized coordinates systems of the different reduced models on the parameters $\boldsymbol{p}$. Issues like mode crossing and veering might arise to which some authors proposed interesting solutions. In [14] a congruence transformation that is compatible with the reduced states is used to address the problem. In [15] a more refined approach is developed in which several different ROM are evaluated and used to create a congruence transformations for each parameter realization. Regardless of the available PMOR technique adopted, the accurate selection of several $\boldsymbol{p}_{\boldsymbol{i}}$ realizations (sampling) for the local reduced models is still an open issue. The topic is addressed in e.g. [13] and present in the literature related to POD [18, 19] and BT [10].

The above mentioned strategies could be successfully adopted for design optimization as in [17] but not always for efficient, e.g. real-time, simulation. The methods in [14, 15] would allow for real-time capabilities, but the direct interpolation of reduced models does not take into account that the system dynamics may be highly influenced by the time dependency of the parameters themselves as shown in [20-22]. Given this, the methodologies as such can be used for e.g. time simulation of parametric systems when the parametric time dependency of the system does not play a crucial role for the overall and local system dynamics.

The technique developed in this work allows to solve the specific set of problems of type

$$
M \ddot{x}+K x=B(p(t)) u
$$

in which the system matrices do not depend on the parameters $\boldsymbol{p}$ while the external load location is parameterized by the matrix $\boldsymbol{B}(\boldsymbol{p}(\boldsymbol{t}))$. The theory will be developed to take into account the influence of the implicit or explicit time dependency of $p(t)$ on the solution. In this category fall all the structural dynamics problems in which the location of external loads and boundary conditions is time varying, e.g. gear contact and sliding components. The developed method could potentially be expanded to include Signorini conditions but as such can be directly used in e.g. continuous contact in a penalty formulation framework. To the authors' knowledge, a similar approach, in which the dynamic parameter dependency is included in a PMOR context and fulfills an energetic principle, has not been proposed in literature and underlines further restriction on classic PMOR approaches. 


\subsection{Stress recovery using reduced order models}

Section 1.1 introduced the concept of MOR via a set of shape vectors in order to increase efficiency in the computation of the displacement fields and, more difficult, reaction forces in structural dynamics and FMBS problems. The main complexity of this approach lies in the selection of an appropriate set of shape vectors [23]. This is especially true when the user is not only interested in having a satisfying approximation of the displacement field but also of strains and internal forces, i.e. stresses [24]. The differential nature of these variables makes them one order less accurate with respect to the displacement field. In MOR based on FE and FMBS, two equivalent ways of computing stress fields directly from the ROM are possible. In the first case, which is also the one followed in this work, the displacement field is known and the stress field can be computed in a post processing step, by using the FE shape functions, as follows:

$$
\begin{gathered}
\boldsymbol{\epsilon}(\boldsymbol{r}, t)=\boldsymbol{D}_{\boldsymbol{\epsilon}} \boldsymbol{x}(\boldsymbol{r}, t) \cong \tilde{\boldsymbol{\epsilon}}(\boldsymbol{r}, t)=\boldsymbol{D}_{\boldsymbol{\epsilon}} \tilde{\boldsymbol{x}}(\boldsymbol{r}, t)=\left[\begin{array}{ccc}
\partial / \partial x & 0 & 0 \\
0 & \partial / \partial y & 0 \\
0 & 0 & \partial / \partial z \\
\partial / \partial y & \partial / \partial x & 0 \\
\partial / \partial z & 0 & \partial / \partial x \\
0 & \partial / \partial z & \partial / \partial y
\end{array}\right]\left[\begin{array}{c}
\tilde{x}_{x} \\
\tilde{x}_{y} \\
\tilde{x}_{z}
\end{array}\right] ; \\
\boldsymbol{\sigma}(\boldsymbol{r}, t) \cong \tilde{\boldsymbol{\sigma}}(\boldsymbol{r}, t)=\boldsymbol{H} \tilde{\boldsymbol{\epsilon}}(\boldsymbol{r}, t)
\end{gathered}
$$

where $\boldsymbol{\epsilon}(\boldsymbol{r}, t)$ is the vector of strain components of the flexible body evaluated at the position $\boldsymbol{r}$ at time $t ; \sigma$ is the corresponding stress field, $\tilde{x}_{x}$ is the nodal displacement in the direction $x$ evaluated at point $\boldsymbol{r}, \boldsymbol{H}$ is the material constitutive matrix and the quantities surmounted by a tilde represent the approximation that is made by using the ROM for the corresponding quantity.

Alternatively, stress shapes can be computed a priori as suggested e.g. in [25]. These shapes represent the stress field that is obtained if the displacement field of each of the shape vectors in $V$ is imposed to the flexible body. The global stress field can then be computed by superposition of the contribution of each stress shape

$$
\begin{gathered}
\tilde{\boldsymbol{\epsilon}}(\boldsymbol{r}, t)=\boldsymbol{D}_{\boldsymbol{\epsilon}} \tilde{\boldsymbol{x}}(\boldsymbol{r}, t)=\boldsymbol{D}_{\boldsymbol{\epsilon}} \boldsymbol{V}(\boldsymbol{r}) \boldsymbol{x}_{\boldsymbol{r}}(t) ; \\
\tilde{\boldsymbol{\sigma}}(\boldsymbol{r}, t)=\boldsymbol{H} \boldsymbol{D}_{\boldsymbol{\epsilon}} \boldsymbol{V}(\boldsymbol{r}) \boldsymbol{x}_{\boldsymbol{r}}(t) .
\end{gathered}
$$

where $D_{\epsilon} V(r)=V_{\epsilon}(r)$ and $H D_{\epsilon} V(r)=V_{\sigma}(r)$ represent respectively a matrix of strain and stress shapes evaluated at the point $\boldsymbol{r}$.

A matrix $\boldsymbol{V}$ consisting of eigenmodes only does not guarantee accurate estimation of internal forces and is therefore not recommended for this use [6,24,25]. Even by inclusion of a high number of such shape vectors, the convergence of the stress field is very slow and presents typical Gibbs like phenomena [24] occurring in the vicinity of the boundary conditions that cannot be met exactly by the eigenmodes (for example in the case of a moving load or boundary condition or a joint in a multibody system). If instead the matrix $\boldsymbol{V}$ includes a complete set of shape vectors capable of meeting the dynamic boundary condition for each I/O point, the convergence of the stress field is highly improved [25]. Such a set of shape vectors can be based on static modes, frequency response modes or Krylov vectors of different orders evaluated at different discrete relevant frequencies. Note that the first two suggested categories are subsets of the larger category of Krylov vectors. They are respectively the zero order Krylov vectors computed at $0 \mathrm{~Hz}$ and at some chosen discrete frequencies. 


\subsection{Contributions and structure}

In the present work the goal is to develop a novel PMOR scheme to efficiently solve problems in the form of eq. 8 potentially leading to real-time performance. The proposed scheme is developed to take into account dynamic parameter variability. The equations of motion are derived by including the parameter dependency directly in the Galerkin projection. Equivalently the same result can be obtained starting from the Lagrange equations in parametric form. This approach yields a compact parametric reduced order model (PROM), at the price of evaluating some extra terms in the equations of motion. A physical interpretation of these terms is given and their importance for the case of fast moving external loads or boundary conditions is analyzed. A special focus is directed towards dynamic stress recovery.

The remainder of the paper is structured as follows. Section 2 covers the selection of shape vectors with the desirable one-to-one relation with respect to the $\mathrm{I} / \mathrm{O}$ points. A rigorous derivation of the parametric equations of motion both for the case of implicit and explicit parameter timedependency is developed. Some consideration on physical interpretations and efficiency are presented. In section 3 several numerical examples with explicit parameter time-dependency are presented. A series of dynamic simulations with different spatial frequency of predefined load distributions is performed. Results show the importance of the dynamic parametric treatment and highlights accurate results both in the displacement and stress fields as well as in transient situations. Section 4 summarizes the main results obtained and proposes further extensions and possible applications of the developed framework.

\section{Time dependent parametric model order reduction scheme with focus on dynamic stress recovery}

\subsection{Choice of reduction space}

As a first step for the development of the proposed approach a set of shape vectors with desirable characteristics needs to be selected to create the ROM. Equation 8 implicitly states that the parameters $\boldsymbol{p}$ defining the load locations are known during the simulation. In order to apply the proposed approach, the reduction space $\boldsymbol{V}$ should reflect the parameter dependency as follows:

$$
\begin{gathered}
\boldsymbol{V}=\boldsymbol{V}(\boldsymbol{p})=\left[\begin{array}{ll}
\boldsymbol{\Phi} & \boldsymbol{\Psi}(\boldsymbol{p})
\end{array}\right], \\
\boldsymbol{\Psi}(\boldsymbol{p})=\left[\begin{array}{llll}
\psi_{1}\left(p_{1}\right) & \psi_{2}\left(p_{2}\right) & \ldots & \psi_{n}\left(p_{n}\right)
\end{array}\right] .
\end{gathered}
$$

In eq.13 the matrix $\boldsymbol{\Phi}$ contains shape vectors that are not parameterized, e.g. eigenmodes of the structure. $\Psi(\boldsymbol{p})$ contains blocks of one or more shape vectors $\psi_{i}\left(p_{i}\right)$ that are directly linked to a single parameter $p_{i}$, as underlined in eq.14. This parameter directly expresses the location of the $i^{\text {th }}$ external load or boundary condition. A set of residual attachment modes or linear combinations of them is a possible choice of $\boldsymbol{\Psi}(\boldsymbol{p})$. The dependency of $\boldsymbol{V}$ on $\boldsymbol{p}$ is implied in the remainder of the work unless necessary to simplify the notation.

To clarify the concept of one-to-one relation between shape vectors and load location further, an example can be instrumental. Let us assume, without loss of generality, that $\boldsymbol{\Phi}$ includes the first few eigenmodes of the structure under analysis, e.g. the cantilever beam in fig.1. $\Psi(\boldsymbol{p})$ can be built by including residual attachment modes. With reference to fig. 1 it can be understood that, given $n$ multiple external point load vectors $\boldsymbol{F}_{i}$, it is generally possible to define $n$ different parameters $p_{i}$ expressing the load locations. This can be done e.g. by using the relative position between the nodes of the structure within which the load is situated. A single attachment mode 


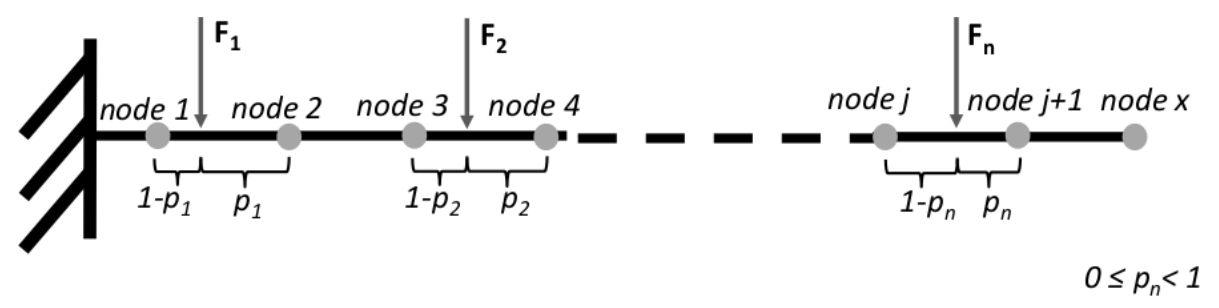

Figure 1: load location and parameterization for a linear interpolation

can be defined for each external load $\boldsymbol{F}_{i}$ and made residual with respect to each of the eigenmodes $\boldsymbol{\Phi}$ by a mass orthonormalization procedure $[4,5]$. If $\boldsymbol{F}_{i}$ is located among two or more nodes, the corresponding residual attachment mode is obtained by linear combination of the residual attachment modes corresponding to the nodes adjacent to the load. The FE shape functions are used as interpolating functions between these shape vectors.

If e.g. linear shape functions are used, the residual attachment modes for each location can be written as

$$
\left\{\begin{array}{c}
\boldsymbol{\psi}_{1}\left(p_{1}\right)=\left(1-p_{1}\right) \boldsymbol{\psi}_{1}^{\text {node } 1}+p_{1} \boldsymbol{\psi}_{1}^{\text {node } 2} \\
\boldsymbol{\psi}_{2}\left(p_{2}\right)=\left(1-p_{2}\right) \boldsymbol{\psi}_{2}^{\text {node } 3}+p_{2} \boldsymbol{\psi}_{2}^{\text {node } 4} \\
\cdots \\
\boldsymbol{\psi}_{n}\left(p_{n}\right)=\left(1-p_{n}\right) \boldsymbol{\psi}_{n}^{\text {nodej }}+p_{n} \boldsymbol{\psi}_{n}^{\text {node }+1}
\end{array}\right.
$$

where $\psi_{n}^{\text {nodej }}$ is the residual attachment mode computed by applying a unit load on node j. To further generalize the concept, in case quadratic shape functions are used in the original FE description (e.g. when using eight noded quadrilater elements) with a load applied to the element edges, eq. 15 would take the form:

$$
\left\{\begin{array}{c}
\boldsymbol{\psi}_{1}\left(p_{1}\right)=0.5\left(p_{1}^{2}-p_{1}\right) \boldsymbol{\psi}_{1}^{\text {node } 1}+\left(1-p_{1}^{2}\right) \boldsymbol{\psi}_{1}^{\text {node } 2}+0.5\left(p_{1}^{2}+p_{1}\right) \boldsymbol{\psi}_{1}^{\text {node } 3} \\
\boldsymbol{\psi}_{2}\left(p_{2}\right)=0.5\left(p_{2}^{2}-p_{2}\right) \boldsymbol{\psi}_{2}^{\text {node } 3}+\left(1-p_{2}^{2}\right) \boldsymbol{\psi}_{2}^{\text {node } 4}+0.5\left(p_{2}^{2}+p_{2}\right) \boldsymbol{\psi}_{2}^{\text {node } 5} \\
\ldots \\
\boldsymbol{\psi}_{n}\left(p_{n}\right)=0.5\left(p_{n}^{2}-p_{2}\right) \boldsymbol{\psi}_{n}^{\text {nodej-1 }}+\left(1-p_{n}^{2}\right) \boldsymbol{\psi}_{n}^{\text {nodej }}+0.5\left(p_{n}^{2}+p_{n}\right) \boldsymbol{\psi}_{n}^{\text {nodej }+1}
\end{array}\right.
$$

This procedure allows to write $\boldsymbol{V}$ in the form of eqs.13-14. In this example, $\boldsymbol{V}$ includes the first eigenmodes of the structure and the residual attachment modes parameterized with respect to the load location by using the parameters $p_{n}$ and the FE shape functions for interpolation. A general procedure to obtain $\boldsymbol{\Phi}$ and the $\psi_{j}^{n o d e j}$ is presented in $[4,5]$. Nonetheless there exist MOR techniques that allow for direct use of the interpolation in eq.15-16. Among others we cite here:

- Eigenmodes + residual attachment modes: here $\Phi$ includes the first eigenmodes of the structure and $\psi_{n}^{\text {nodej }}$ are single residual attachment modes pre-computed for each possible loading location. This set is statically complete, i.e. it is capable of representing the same static deformation of the original FE model for each load location [3]. This approach is used in the remainder of the work;

- Eigenmodes + residual FRM: here $\boldsymbol{\Phi}$ includes the first eigenmodes of the structure and $\psi_{n}^{\text {nodej }}$ are one or more residual FRM pre-computed for each possible loading location and 
for a number of discrete frequencies of interest. This set is statically complete provided that $0 \mathrm{~Hz}$ FRM are included and shows high accuracy around the chosen discrete frequencies;

- Krylov vectors: here $\boldsymbol{\Phi}$ is null and $\psi_{n}^{\text {nodej }}$ includes one or more Krylov vectors for each possible loading location for different orders evaluated at different frequencies (expansion points). This set is statically complete provided that $0 \mathrm{~Hz}$ is chosen as one of the expansion points. FRM and attachment modes can be seen as a particular subset of Krylov vectors [25].

The above discussion allows to construct a reduction space $\boldsymbol{V}$ that is, at least partially, directly linked to the load locations through a set of parameters. Moreover it is interesting to notice that by following the steps in $[4,5]$ it is always possible to retain the one-to-one relation but also to make sure that $\boldsymbol{\Phi}$ and $\boldsymbol{\Psi}(\boldsymbol{p})$ remain mass and stiffness orthogonal,

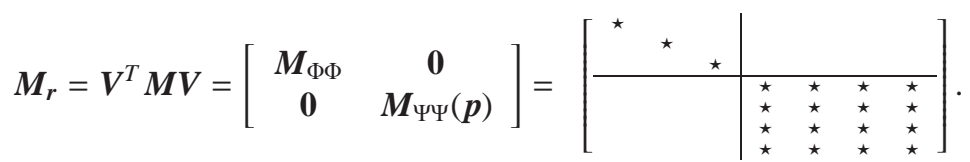

Here $M_{r}$ is a block diagonal matrix and the stars indicate its desirable sparsity pattern. Given the above discussion, the nodal coordinates can be written in the following parameterized form as

$$
x \cong \tilde{x}=V x_{r}=\left[\begin{array}{ll}
\Phi & \Psi(p)
\end{array}\right]\left[\begin{array}{l}
x_{\Phi} \\
x_{\Psi}
\end{array}\right] .
$$

As a remark, any interpolation that makes use of FE shape functions is meaningful in the developed framework and can be used to interpolate the set of shape vectors chosen. This is to say that some important properties like mass and stiffness orthogonality of the interpolated shape vectors $\boldsymbol{\Psi}(\boldsymbol{p})$ with respect to $\boldsymbol{\Phi}$ are preserved. Moreover in the particular case treated, where only the external load is parameterized, mode veering and crossing problems will not arise and a mode tracking technique is inherently used to perform the interpolation among the correct shape vectors to obtain $\Psi(\boldsymbol{p})$. These characteristics are not necessarily fulfilled by other interpolation methods. A very general procedure that allows to maintain many desirable properties of the interpolated vector spaces is found in [26]. This method, allows to interpolate any type of reduced matrices and reduction spaces even when the physical meaning of the generalized coordinates is not kept between different parameter realizations. An extension of the current work to more general time dependent PMOR strategies could benefit from such an interpolation approach.

The presented work focuses on 2 dimensional problems for which the interpolation of each of the vectors in eq.15-16 depends on a single parameter. Considering loads moving on 3 dimensional curved surfaces would induce some additional complexity. The applicability of the proposed method to more complex example cases is currently under evaluation. Preliminary results that extend the generality of the approach to a more general framework are promising.

\subsection{Time dependent parametric model order reduction}

Once the proper set of shape vectors $\boldsymbol{V}$ and reduced coordinates $\boldsymbol{x}_{\boldsymbol{r}}$ are defined, the nodal velocity and acceleration vector can be expressed by time derivation as:

$$
\dot{x} \cong \dot{\tilde{x}}=V \dot{x}_{r}+\dot{V} x_{r}=\left[\begin{array}{ll}
\Phi & \Psi(p)
\end{array}\right]\left[\begin{array}{c}
\dot{x}_{\Phi} \\
\dot{x}_{\Psi}
\end{array}\right]+\left[\begin{array}{cc}
\dot{\Phi} & \dot{\Psi}(p, \dot{p})
\end{array}\right]\left[\begin{array}{c}
x_{\Phi} \\
x_{\Psi}
\end{array}\right]
$$




$$
\begin{gathered}
\ddot{\boldsymbol{x}} \cong \ddot{\tilde{x}}=V \ddot{x}_{r}+2 \dot{V} \dot{\boldsymbol{x}}_{r}+\ddot{\boldsymbol{V}} x_{r}= \\
=\left[\begin{array}{ll}
\boldsymbol{\Phi} & \Psi(p)
\end{array}\right]\left[\begin{array}{c}
\ddot{x}_{\Phi} \\
\ddot{x}_{\Psi}
\end{array}\right]+2\left[\begin{array}{ll}
\dot{\Phi} & \dot{\Psi}(p, \dot{p})
\end{array}\right]\left[\begin{array}{c}
\dot{x}_{\Phi} \\
\dot{x}_{\Psi}
\end{array}\right]+\left[\begin{array}{ll}
\ddot{\Phi} & \ddot{\Psi}(p, \dot{p}, \ddot{p})
\end{array}\right]\left[\begin{array}{c}
x_{\Phi} \\
x_{\Psi}
\end{array}\right] .
\end{gathered}
$$

The second term on the right hand side (RHS) of eq.19 and the last two terms of eq.20 are normally not present when deriving the equations of motion of an FE model. Their physical meaning is inherent with the time variability of the reduction space $V$ given by a dynamic change in $\boldsymbol{p}(t)$. In order to simplify the notation, the dependency of $\boldsymbol{\Psi}, \dot{\boldsymbol{\Psi}}$ and $\ddot{\boldsymbol{\Psi}}$ on $\boldsymbol{p}, \dot{\boldsymbol{p}}$ and $\ddot{\boldsymbol{p}}$ is implied in the remainder unless clarity requires its full notation. By noticing that $\boldsymbol{\Phi}$ is a constant matrix and by simple matrix manipulation it is possible to write:

$$
\begin{aligned}
& \dot{\Phi}=0 ;
\end{aligned}
$$

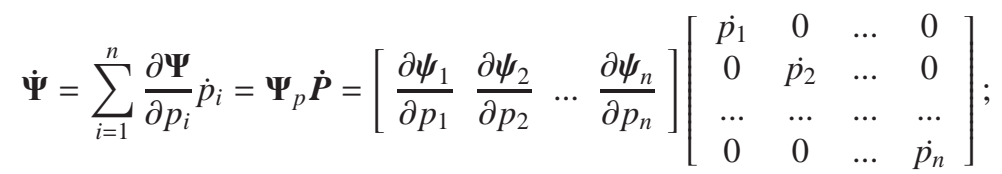

$$
\begin{aligned}
& \ddot{\boldsymbol{\Psi}}=\boldsymbol{\Psi}_{p} \ddot{\boldsymbol{P}}+\sum_{i=1}^{n} \frac{\partial \boldsymbol{\Psi}_{p}}{\partial p_{i}} \dot{p}_{i} \dot{\boldsymbol{P}}=\boldsymbol{\Psi}_{p} \ddot{\boldsymbol{P}}+\boldsymbol{\Psi}_{p p} \dot{\boldsymbol{P}}^{2}= \\
& =\boldsymbol{\Psi}_{p}\left[\begin{array}{cccc}
\ddot{p_{1}} & 0 & \ldots & 0 \\
0 & \ddot{p_{2}} & \ldots & 0 \\
\ldots & \ldots & \ldots & \ldots \\
0 & 0 & \ldots & \ddot{p_{n}}
\end{array}\right]+\left[\begin{array}{llll}
\frac{\partial^{2} \boldsymbol{\psi}_{1}}{\partial p_{1}{ }^{2}} & \frac{\partial^{2} \boldsymbol{\psi}_{2}}{\partial p_{2}{ }^{2}} & \ldots & \frac{\partial^{2} \boldsymbol{\psi}_{n}}{\partial p_{n}{ }^{2}}
\end{array}\right]\left[\begin{array}{cccc}
\dot{p}_{1}{ }^{2} & 0 & \ldots & 0 \\
0 & \dot{p_{2}} & \ldots & 0 \\
\ldots & \ldots & \ldots & \ldots \\
0 & 0 & \ldots & \dot{p}_{n}{ }^{2}
\end{array}\right] \text {, }
\end{aligned}
$$

where generally $\boldsymbol{\Psi}_{p}$ depends on the parameters $\boldsymbol{p}$. If the linear interpolation in eq.15 is used, then $\boldsymbol{\Psi}_{p}=$ const and $\boldsymbol{\Psi}_{p p}=0$. This is not true for higher order interpolations like in eq.16. The parameter vector $\boldsymbol{p}$ can be an explicit function of time $\boldsymbol{p}(t)$ or an implicit function $\boldsymbol{p}(\boldsymbol{q}(t))$ where $\boldsymbol{q}$ can represent e.g. the generalized coordinates of a controlled mechanism attached to the structure under analysis or the controller states themselves. The cases in which $\boldsymbol{p}=\boldsymbol{p}\left(\boldsymbol{x}_{\boldsymbol{r}}(t)\right)$ and of $\psi_{n}=\psi_{n}\left(p_{1}, p_{2}, \ldots, p_{n}\right) \neq \psi_{n}\left(p_{n}\right)$ are not treated in the remainder of the work. These cases can be relevant e.g. in the development of a similar PMOR strategy for non-linear flexible multibody systems. While conceptually the development of the method remains similar, the derivation of the equations of motion becomes considerably more involved.

Substituting eqs.19-23 into eq.8 and pre-multiplying by $\boldsymbol{V}^{T}$ we obtain:

$$
\boldsymbol{V}^{T} \boldsymbol{M}\left[\boldsymbol{V} \ddot{\boldsymbol{x}}_{r}+2 \dot{\boldsymbol{V}} \dot{\boldsymbol{x}}_{r}+\ddot{\boldsymbol{V}} \boldsymbol{x}_{r}\right]+\boldsymbol{V}^{T} \boldsymbol{K} \boldsymbol{V} \boldsymbol{x}_{r}=\boldsymbol{M}_{\boldsymbol{V} V} \ddot{\boldsymbol{x}}_{r}+2 \boldsymbol{M}_{\boldsymbol{V} \dot{V}} \dot{\boldsymbol{x}}_{r}+\left(\boldsymbol{K}_{V V}+\boldsymbol{M}_{V \ddot{V}}\right) \boldsymbol{x}_{r}=\boldsymbol{V}^{T} \boldsymbol{B} \boldsymbol{u}=\boldsymbol{B}_{r} \boldsymbol{u}
$$

where:

$$
\begin{gathered}
\boldsymbol{M}_{V V}=\boldsymbol{M}_{\boldsymbol{r}}=\left[\begin{array}{cc}
\boldsymbol{M}_{\Phi \Phi} & \mathbf{0} \\
\mathbf{0} & \boldsymbol{M}_{\Psi \Psi}(\boldsymbol{p})
\end{array}\right]=\left[\begin{array}{cc}
\boldsymbol{\Phi}^{T} \boldsymbol{M \Phi} & \mathbf{0} \\
\mathbf{0} & \boldsymbol{\Psi}^{T} \boldsymbol{M \Psi}
\end{array}\right] \\
\boldsymbol{M}_{V \dot{V}}=\left[\begin{array}{cc}
\mathbf{0} & \mathbf{0} \\
\mathbf{0} & \boldsymbol{M}_{\Psi \Psi_{p}}(\boldsymbol{p}) \dot{\boldsymbol{P}}
\end{array}\right]=\left[\begin{array}{cc}
\mathbf{0} & \mathbf{0} \\
\mathbf{0} & \boldsymbol{\Psi}^{T} \boldsymbol{M} \boldsymbol{\Psi}_{p} \dot{\boldsymbol{P}}
\end{array}\right] \\
\boldsymbol{M}_{V \ddot{V}}=\left[\begin{array}{cc}
\mathbf{0} & \mathbf{0} \\
\mathbf{0} & \boldsymbol{M}_{\Psi \Psi_{p}}(\boldsymbol{p}) \ddot{\boldsymbol{P}}+\boldsymbol{M}_{\Psi \Psi_{p p}}(\boldsymbol{p}) \dot{\boldsymbol{P}}^{2}
\end{array}\right]=\left[\begin{array}{ccc}
\mathbf{0} & \mathbf{0} \\
\mathbf{0} & \boldsymbol{\Psi}^{T} \boldsymbol{M} \boldsymbol{\Psi}_{p} \ddot{\boldsymbol{P}}
\end{array}\right]+\left[\begin{array}{cc}
\mathbf{0} & \mathbf{0} \\
\mathbf{0} & \boldsymbol{\Psi}^{T} \boldsymbol{M} \boldsymbol{\Psi}_{p p} \dot{\boldsymbol{P}}^{2}
\end{array}\right] ;
\end{gathered}
$$




$$
\boldsymbol{K}_{\boldsymbol{V} V}=\boldsymbol{K}_{\boldsymbol{r}}=\left[\begin{array}{cc}
\boldsymbol{K}_{\Phi \Phi} & \mathbf{0} \\
\mathbf{0} & \boldsymbol{K}_{\Psi \Psi}(\boldsymbol{p})
\end{array}\right]=\left[\begin{array}{cc}
\boldsymbol{\Phi}^{T} \boldsymbol{K} \boldsymbol{\Phi} & \mathbf{0} \\
\mathbf{0} & \boldsymbol{\Psi}^{T} \boldsymbol{K} \Psi
\end{array}\right] .
$$

Equations 25-28 have been obtained by exploiting eq. 21 and the following desirable properties of the submatrices involved:

$$
\begin{aligned}
& \boldsymbol{\Phi}^{T} \boldsymbol{M} \boldsymbol{\Psi}=\mathbf{0}=\boldsymbol{\Psi}^{T} \boldsymbol{M} \boldsymbol{\Phi} \\
& \boldsymbol{\Phi}^{T} \boldsymbol{K} \Psi=\mathbf{0}=\boldsymbol{\Psi}^{T} \boldsymbol{K} \boldsymbol{\Phi} ; \\
& \Psi_{p}{ }^{T} \boldsymbol{M \Phi}=\mathbf{0}=\boldsymbol{\Phi}^{T} \boldsymbol{M} \boldsymbol{\Psi}_{\boldsymbol{p}} ; \\
& \boldsymbol{\Psi}_{\boldsymbol{p} \boldsymbol{p}}{ }^{T} \boldsymbol{M \Phi}=\mathbf{0}=\boldsymbol{\Phi}^{T} \boldsymbol{M} \boldsymbol{\Psi}_{\boldsymbol{p p}} .
\end{aligned}
$$

Equations 29 and 30 state the mass ans stiffness orthogonality between $\boldsymbol{\Phi}$ and $\boldsymbol{\Psi}$ that is a necessary characteristic of the reduction space $V$ and is imposed by construction, see eq.17. Equations 31 and 32 follow from eqs.15,16 and 29 and are generally valid for any interpolation that is linear with respect to $\psi_{n}^{n o d e j}$ but can be non-linear in $p_{n}$. Summarizing, the equations of motion can finally be written as

$$
M_{V V} \ddot{x}_{r}+2 M_{V \dot{V}} \dot{x}_{r}+\left(K_{V V}+M_{V \ddot{V}}\right) x_{r}=B_{r} u,
$$

or, highlighting the parameter dependency, as

$$
\begin{aligned}
& {\left[\begin{array}{cc}
\boldsymbol{M}_{\Phi \Phi} & \mathbf{0} \\
\mathbf{0} & \boldsymbol{M}_{\Psi \Psi}(\boldsymbol{p})
\end{array}\right] \ddot{\boldsymbol{x}}_{\boldsymbol{r}}+2\left[\begin{array}{cc}
\mathbf{0} & \mathbf{0} \\
\mathbf{0} & \boldsymbol{M}_{\Psi \Psi_{p}}(\boldsymbol{p}) \dot{\boldsymbol{P}}
\end{array}\right] \dot{\boldsymbol{x}}_{\boldsymbol{r}}+} \\
& +\left(\left[\begin{array}{cc}
\boldsymbol{K}_{\Phi \Phi} & \mathbf{0} \\
\mathbf{0} & \boldsymbol{K}_{\Psi \Psi}(\boldsymbol{p})+\boldsymbol{M}_{\Psi \Psi_{p}}(\boldsymbol{p}) \ddot{\boldsymbol{P}}+\boldsymbol{M}_{\Psi \Psi_{p p}}(\boldsymbol{p}) \dot{\boldsymbol{P}}^{2}
\end{array}\right]\right) \boldsymbol{x}_{\boldsymbol{r}}=\boldsymbol{B}_{r} \boldsymbol{u} .
\end{aligned}
$$

This equation has been derived by using Galerkin projections. It can be shown that the same result could be obtained starting from the Lagrange equations written in parametric form:

$$
\frac{d}{d t} \frac{\partial \tilde{\mathcal{L}}}{\partial \dot{\boldsymbol{x}}_{\boldsymbol{r}}}-\frac{\partial \tilde{\mathcal{L}}}{\partial \boldsymbol{x}_{\boldsymbol{r}}}=\frac{d}{d t} \frac{\partial \tilde{T}}{\partial \dot{\boldsymbol{x}}_{\boldsymbol{r}}}-\frac{\partial \tilde{T}}{\partial \boldsymbol{x}_{\boldsymbol{r}}}+\frac{\partial \tilde{U}}{\partial \boldsymbol{x}_{\boldsymbol{r}}}=\tilde{\boldsymbol{F}}_{\text {ext }}=\boldsymbol{B}_{r} \boldsymbol{u},
$$

where $\tilde{\mathcal{L}}=\tilde{T}-\tilde{U}$ is the Lagrangian of the reduced order model, $\tilde{T}, \tilde{U}$ are the reduced kinetic and potential energy and can be written as:

$$
\begin{array}{r}
T=\frac{1}{2} \dot{\boldsymbol{x}}^{T} \boldsymbol{M} \dot{\boldsymbol{x}} \cong \tilde{T}=\frac{1}{2} \dot{\tilde{\boldsymbol{x}}}^{T} \boldsymbol{M} \dot{\tilde{\boldsymbol{x}}}=\frac{1}{2}\left(\boldsymbol{V} \dot{\boldsymbol{x}}_{\boldsymbol{r}}+\dot{\boldsymbol{V}} \boldsymbol{x}_{\boldsymbol{r}}\right)^{T} \boldsymbol{M}\left(\boldsymbol{V} \dot{\boldsymbol{x}}_{\boldsymbol{r}}+\dot{\boldsymbol{V}} \boldsymbol{x}_{\boldsymbol{r}}\right)= \\
=\frac{1}{2}\left[\dot{\boldsymbol{x}}_{\boldsymbol{r}}^{T} \boldsymbol{M}_{\boldsymbol{V} \boldsymbol{V}} \dot{\boldsymbol{x}}_{\boldsymbol{r}}+2 \dot{\boldsymbol{x}}_{\boldsymbol{r}}^{T} \boldsymbol{M}_{\boldsymbol{V} \dot{\boldsymbol{V}}} \boldsymbol{x}_{\boldsymbol{r}}+\boldsymbol{x}_{\boldsymbol{r}}{ }^{T} \boldsymbol{M}_{\dot{V} \dot{V}} \boldsymbol{x}_{\boldsymbol{r}}\right] \\
U=\frac{1}{2} \boldsymbol{x}^{T} \boldsymbol{K} \boldsymbol{x} \cong \tilde{U}=\frac{1}{2} \tilde{\boldsymbol{x}}^{T} \boldsymbol{K} \tilde{\boldsymbol{x}}=\frac{1}{2}\left(\boldsymbol{V} \boldsymbol{x}_{\boldsymbol{r}}\right)^{T} \boldsymbol{K} \boldsymbol{V} \boldsymbol{x}_{\boldsymbol{r}}=\frac{1}{2} \boldsymbol{x}_{\boldsymbol{r}}{ }^{T} \boldsymbol{K}_{\boldsymbol{V} V} \boldsymbol{x}_{\boldsymbol{r}}
\end{array}
$$

Since eq.34 can be obtained starting from an energy principle then the equations of motion preserve the energy in the system. The terms $\boldsymbol{M}_{\Psi \Psi_{p}}(\boldsymbol{p}) \dot{\boldsymbol{P}} \dot{\boldsymbol{x}}_{\boldsymbol{\Psi}}$ and $\left(\boldsymbol{M}_{\Psi \Psi_{p}}(\boldsymbol{p}) \ddot{\boldsymbol{P}}+\boldsymbol{M}_{\Psi \Psi_{p p}}(\boldsymbol{p}) \dot{\boldsymbol{P}}^{2}\right) \boldsymbol{x}_{\boldsymbol{\Psi}}$ in eq.34 are not taken into account in [14] and [15]. However it should be pointed out that those methods are not developed for systems with time-varying parameters and remain consistent in their frameworks.

These extra terms account for variations of the reduced stiffness and reduced mass and are necessary to provide a correct repartition of kinetic and potential energy when time varying parameters are present. In general these terms would not sum up to zero. It is clear that matrix 
interpolation approaches are not energy-preserving unless $\dot{\boldsymbol{P}}=0$ and $\ddot{\boldsymbol{P}}=0$, that is when the parameter variation happens statically. The method can still be used safely for design optimization, where the parameter $\boldsymbol{p}$ does not vary in time as in [14] and is likely to deliver good approximations for slow parameter variation. Care should be taken in dynamic cases.

It is also important to highlight that in the more involved case in which $\boldsymbol{p}=\boldsymbol{p}\left(\boldsymbol{x}_{\boldsymbol{r}}(t)\right)$, the parametric equations of motion should be derived starting from Lagrange equations and a simple Galerkin projection is not sufficient to ensure energy preservation.

It should also be observed that, in the developed framework, the parameterization is exclusively related to the external load location. In this case the system dynamics of the unreduced model in eq. 8 are not affected by the parameterization. Conceptually this should not be seen as a limitation of the approach since a very similar relation to the one in eq. 34 could be easily obtained if e.g. time varying position-level boundary conditions would exist. In this case the unreduced system dynamics would be affected by the parameterization. The above interesting cases will be the main focus of future works.

A further observation concerns the a priori knowledge of $\boldsymbol{p}(t)$. In the case that $\boldsymbol{p}(t)$ is known, then $\dot{\boldsymbol{P}}$ and $\ddot{\boldsymbol{P}}$ can be precomputed. Moreover, all the parameter dependent matrices in eq.34 and their inverses, if needed, can also be pre-computed. By doing so, the computational speed is drastically increased and the PMOR scheme becomes very compact and efficient to implement. Nonetheless, the above mentioned case is not general. If $\boldsymbol{p}=\boldsymbol{p}(\boldsymbol{q}(t))$ and $\boldsymbol{q}(t)$ is not known a priori, then $\dot{\boldsymbol{P}}$ and $\ddot{\boldsymbol{P}}$ need to be computed, during time simulation as follows:

$$
\begin{gathered}
\dot{\boldsymbol{P}}=\left[\begin{array}{cccc}
p_{1} & 0 & \ldots & 0 \\
0 & \dot{p_{2}} & \ldots & 0 \\
\ldots & \ldots & \ldots & \ldots \\
0 & 0 & \ldots & \dot{p_{n}}
\end{array}\right]=\left[\begin{array}{cccc}
\left(\partial p_{1} / \partial \boldsymbol{q}\right) \dot{\boldsymbol{q}} & 0 & \ldots & 0 \\
0 & \left(\partial p_{2} / \partial \boldsymbol{q}\right) \dot{\boldsymbol{q}} & \ldots & 0 \\
\ldots & \ldots & \ldots & \ldots \\
0 & 0 & \ldots & \left(\partial p_{n} / \partial \boldsymbol{q}\right) \dot{\boldsymbol{q}}
\end{array}\right]=\operatorname{diag}\left(\left[\boldsymbol{P}_{q} \dot{\boldsymbol{q}}\right]_{i}\right) ; \\
\ddot{\boldsymbol{P}}=\left[\begin{array}{ccccc}
\ddot{p_{1}} & 0 & \ldots & 0 \\
0 & \ddot{p}_{2} & \ldots & 0 \\
\ldots & \ldots & \ldots & \ldots \\
0 & 0 & \ldots & \ddot{p}_{n}
\end{array}\right]=\operatorname{diag}\left(\left[\boldsymbol{P}_{q} \ddot{\boldsymbol{q}}\right]_{i}\right)+\operatorname{diag}\left(\left[\sum_{i=1}^{l}\left[\boldsymbol{P}_{q_{q} \dot{q}_{i}} \dot{q}_{i} \dot{\boldsymbol{q}}\right]_{i}\right),\right.
\end{gathered}
$$

where $\boldsymbol{P}_{\boldsymbol{q}}$ is the Jacobian of the parameters vector $\boldsymbol{p}, \boldsymbol{P}_{\boldsymbol{q} q_{i}}$ is the derivative of the Jacobian matrix $\boldsymbol{P}_{\boldsymbol{q}}$ with respect to $q_{i}$, and $l$ is the number of $q_{i}$.

Evaluating the terms in eq.39 for large sizes of $\boldsymbol{q}$ and $\boldsymbol{p}$ can become numerically expensive. Moreover the parameter dependent matrices in eq.34 are highly non-linear and cannot be precomputed. As an example, for a given $\boldsymbol{p}$ realization, building the matrix $\boldsymbol{M}_{\Psi \Psi_{p}}(\boldsymbol{p})$ involves the multiplication of large matrices. The numerical complexity is of order $O\left(n x^{2}+x n^{2}\right)$ multiplications where $n$ is the amount of parameterized shape vectors and $x$ is the number of DOF in the original FE model that can be prohibitively large. As a solution to this problem, it is possible to exploit the polynomial relation in eq.15 or eq.16. Depending on the interpolation type, it is generally possible to write the matrices in eq.34, e.g. $\boldsymbol{M}_{\Psi \Psi_{p}}(\boldsymbol{p})$, as a sum of matrices that can be precomputed and in which the parametric dependency is separable. To clarify this concept, let us write this relation for the simple case of linear interpolation in eq.15:

$$
\boldsymbol{M}_{\Psi \Psi}(\boldsymbol{p})=\boldsymbol{\Psi}^{T} \boldsymbol{M} \boldsymbol{\Psi}=\left[\begin{array}{lllll}
\psi_{1}\left(p_{1}\right) & \psi_{2}\left(p_{2}\right) & \ldots & \boldsymbol{\psi}_{n}\left(p_{n}\right)
\end{array}\right]^{T} \boldsymbol{M}\left[\begin{array}{lllll}
\boldsymbol{\psi}_{1}\left(p_{1}\right) & \psi_{2}\left(p_{2}\right) & \ldots & \psi_{n}\left(p_{n}\right)
\end{array}\right] ;
$$


by using eq. 15 and reformulating

$$
\begin{gathered}
{\left[\begin{array}{cccc}
\boldsymbol{\psi}_{1}^{\text {node } 1} & \boldsymbol{\psi}_{2}^{\text {node } 3} & \ldots & \boldsymbol{\psi}_{n}^{\text {nodej }}
\end{array}\right]=\boldsymbol{\Psi}^{j},} \\
{\left[\begin{array}{cccc}
\boldsymbol{\psi}_{1}^{\text {node } 2} & \boldsymbol{\psi}_{2}^{\text {node } 4} & \ldots & \boldsymbol{\psi}_{n}^{\text {node } j+1}
\end{array}\right]=\boldsymbol{\Psi}^{j+1},} \\
{\left[\begin{array}{cccc}
\left(1-p_{1}\right) & 0 & \ldots & 0 \\
0 & \left(1-p_{2}\right) & \ldots & 0 \\
\ldots & \ldots & \ldots & \ldots \\
0 & 0 & \ldots & \left(1-p_{n}\right)
\end{array}\right]=[\boldsymbol{I}-\boldsymbol{P}],} \\
{\left[\begin{array}{cccc}
p_{1} & 0 & \ldots & 0 \\
0 & p_{2} & \ldots & 0 \\
\ldots & \ldots & \ldots & \ldots \\
0 & 0 & \ldots & p_{n}
\end{array}\right]=\boldsymbol{P},}
\end{gathered}
$$

it is possible to separate the parametric relationship as

$$
\begin{gathered}
\boldsymbol{M}_{\Psi}(\boldsymbol{p})=[\boldsymbol{I}-\boldsymbol{P}]\left(\boldsymbol{\Psi}^{j}\right)^{T} \boldsymbol{M} \boldsymbol{\Psi}^{j}[\boldsymbol{I}-\boldsymbol{P}]+[\boldsymbol{I}-\boldsymbol{P}]\left(\boldsymbol{\Psi}^{j}\right)^{T} \boldsymbol{M} \boldsymbol{\Psi}^{j+1} \boldsymbol{P}+ \\
+\boldsymbol{P}\left(\boldsymbol{\Psi}^{j+1}\right)^{T} \boldsymbol{M} \boldsymbol{\Psi}^{j}[\boldsymbol{I}-\boldsymbol{P}]+\boldsymbol{P}\left(\boldsymbol{\Psi}^{j+1}\right)^{T} \boldsymbol{M} \boldsymbol{\Psi}^{j+1} \boldsymbol{P}= \\
=[\boldsymbol{I}-\boldsymbol{P}] \boldsymbol{M}_{\boldsymbol{\Psi}^{j} \boldsymbol{\Psi}^{j}}[\boldsymbol{I}-\boldsymbol{P}]+[\boldsymbol{I}-\boldsymbol{P}] \boldsymbol{M}_{\boldsymbol{\Psi}^{j} \boldsymbol{\Psi}^{j+1}} \boldsymbol{P}+\boldsymbol{P} \boldsymbol{M}_{\boldsymbol{\Psi}^{j+1} \boldsymbol{\Psi}^{j}}[\boldsymbol{I}-\boldsymbol{P}]+\boldsymbol{P} \boldsymbol{M}_{\boldsymbol{\Psi}^{j+1} \boldsymbol{\Psi}^{j+1}} \boldsymbol{P} .
\end{gathered}
$$

By using the above formulation, all the matrices of the type $\boldsymbol{M}_{\boldsymbol{\Psi}^{j} \boldsymbol{\Psi}^{j}}$ can be assembled by using a precomputed set of shape vectors so that the complexity of the algorithm significantly decreases to order $O\left(g n^{2}\right)$ multiplications, with $g$ being the small number of matrices that need to be summed in eq.45. The main complexity of the algorithm is related to the availability of $\dot{\boldsymbol{P}}$ and $\ddot{\boldsymbol{P}}$. In case these are not readily available, numerical estimates obtained from finite differentiation could be used, as well as exact derivatives obtained from previous time-steps. These solutions could potentially cause numerical difficulties that will be analyzed in future works.

As a final remark, it should be underlined that at least the same number of shape vectors as there are point loads acting on the structure should be used to preserve static completeness. For this reason the method is, as such, not well suited for problems in which very large moving interfaces are present (e.g. unknown pressure distributions) since the number of time varying shape vectors could become prohibitively large. In the foreseen application to gears and bearings contact problems this is rarely the case especially if a low number of teeth or rolling elements is in contact at a given instant.

\section{Numerical results}

The flow chart in fig. 2 summarizes the computational steps involved in the methodology developed in section 2. These steps include computationally extensive pre-processing steps and lighter on-line evaluations. The procedure has been implemented in Matlab and applied to two different FE models.

\subsection{Example1: Rectangular block with Hertzian-like moving load}

The model is built using eight noded membrane elements with quadratic shape functions. Figure 3 shows the deformed FE mesh. The simplicity of this model is justified by the aim of this work: validate the developed PMOR scheme on a test case that is representative of a larger 


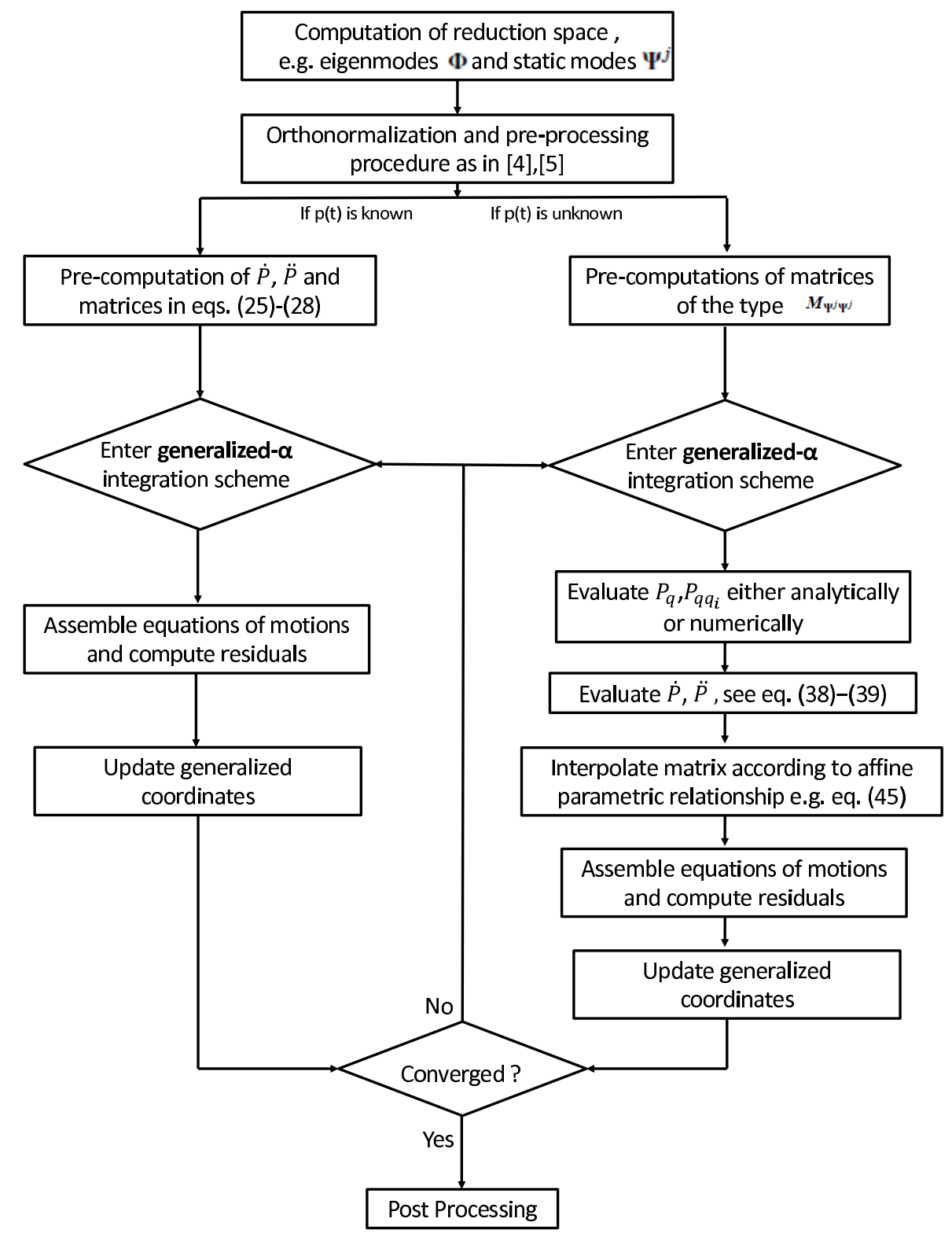

Figure 2: Time-varying PMOR flow chart of computational steps 


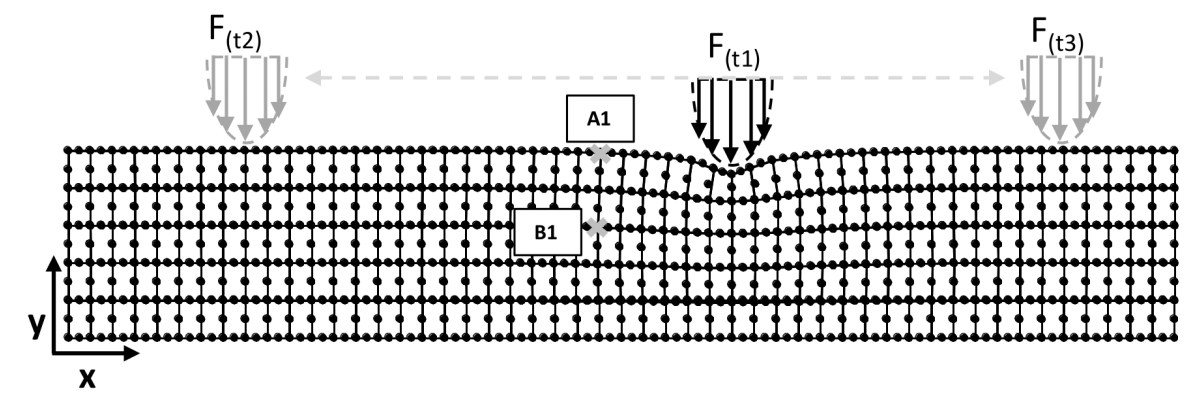

Figure 3: FE model

Table 1: general characteristics of the FE model used for simulation

\begin{tabular}{|c|c|}
\hline density $\left[\mathrm{kg} / \mathrm{m}^{3}\right]$ & 7860 \\
\hline weight $[\mathrm{kg}]$ & 0.982 \\
\hline thickness $[\mathrm{mm}]$ & 10 \\
\hline Young's modulus $[\mathrm{GPa}]$ & 210 \\
\hline Poisson's ratio & 0.3 \\
\hline total number of nodes & $861(1722 \mathrm{DOF})$ \\
\hline number of clamped nodes & $101(202 \mathrm{DOF})$ \\
\hline number of possibly loaded nodes & 81 \\
\hline mode 1 frequency $[\mathrm{kHz}]$ & 14.74 \\
\hline mode 2 frequency $[\mathrm{kHz}]$ & 17.01 \\
\hline
\end{tabular}

class of problems but remains relatively easy to analyze. Moreover given the quadratic nature of the shape functions, all the dynamic terms in eq. 34 are actively contributing to the solution.

The block is clamped at the lower horizontal edge in both $\mathrm{x}$ and $\mathrm{y}$ DOFs. The main characteristics of the model are summarized in table 1 . The time simulation is performed by using the generalized- $\alpha$ numerical scheme [27]. This scheme allows for the introduction of user definable numerical damping and can be used to solve ODEs as well as DAEs up to index-3. This characteristics allow for an easier extension of the proposed approach to constrained multibody simulation. This first example is used to evaluate the proposed PMOR scheme during a dynamic simulation using quadratic interpolation. From fig. 3 it can be seen that a load distribution following a Hertzian-like pattern is acting on the top edge of the block. The load is constantly directed parallel to the $y$-direction. The following expressions define the load location and the parameter used to create the load pattern:

$$
\begin{gathered}
x_{l}=c+x_{\text {mag }} \sin \left(2 \pi f_{i} t\right) \\
p_{1}=\frac{x_{l}-x_{\text {node } j}}{2\left(x_{\text {node }} j+1-x_{\text {node } j-1}\right)}-1 \\
F_{\text {ext }}=F_{\text {amp }} F_{\text {distr }}=F_{\text {amp }}\left(\frac{E}{1-v^{2}}\right)\left(\frac{d^{2}}{a^{2}}\right)^{0.5}\left(\frac{1-r^{2}}{a^{2}}\right)^{0.5} A_{\text {ref }}
\end{gathered}
$$


Table 2: example 1 simulation details

\begin{tabular}{|c|c|}
\hline simulation time $[\mathrm{s}]$ & $0.2 \cdot 10^{-2}$ \\
\hline spectral radius at $\infty \rho_{\infty}$ & 0.1 \\
\hline time step $h[\mathrm{~s}]$ & $0.16 \cdot 10^{-5}$ \\
\hline initial load position $c[\mathrm{~m}]$ & -0.1 (central node) \\
\hline load position amplitude $x_{\operatorname{mag}}[\mathrm{m}]$ & 0.2 \\
\hline load position frequency $f_{1}[\mathrm{~Hz}]$ & $10^{3}$ \\
\hline load maximum amplitude $F_{\max }[\mathrm{N}]$ & $3 \cdot 10^{6}$ \\
\hline load raising time $T_{s}[\mathrm{~s}]$ & $0.2 \cdot 10^{-2}$ \\
\hline penetration parameter $d[\mathrm{~m}]$ & $0.1 \cdot 10^{-2}$ \\
\hline width of loaded region $a[\mathrm{~m}]$ & $0.11 \cdot 10^{-1}$ \\
\hline reference area $A_{\text {ref }}\left[\mathrm{m}^{2}\right]$ & $0.11 \cdot 10^{-4}$ \\
\hline
\end{tabular}

Where the numerical values of the parameters in eqs.46-48 are defined in table 2. Here $d$ is a parameter related to the maximum penetration depth, $a$ is the half width of the loaded region and $r$ is the distance with respect to the center of the load distribution and can vary between $-a$ and $a$. The external load $F_{\text {ext }}$ starts from an amplitude $F_{\text {amp }}=0 \mathrm{~N}$ at time $t_{0}=0 \mathrm{~s}$ and increases linearly up to $F_{\max }$ at the end of the simulation. The continuous function $F_{\text {ext }}$ used to describe the load is approximated with five point loads as depicted in fig. 3 each spaced $0.005 \mathrm{~m}$. The $x$ location of the load is given by the value of $x_{l}$. Equation 46 shows that the load starts from a position $c$ at the midspan of the rectangular block and moves sinusoidally with frequency $f_{i}$ and amplitude $x_{\text {mag }}$. Since a single external load pattern is applied, the parameter vector $\boldsymbol{p}$ reduces to the scalar quantity $p_{1}$ that can vary between -1 and 1 depending on the relative position of the load with respect to the nodes adjacent to it, see eq.47. The simulation parameters are also gathered in table 2. The generalized- $\alpha$ damping parameter $\rho_{\infty}$ is chosen to have a minimal effect in the frequency range of interest and the time step is selected small enough to have at least 10 integration points during the movement of the load along each element edge. The choice of such a conservative time step assures a smooth transition when the load travels from one element to another and allows precise evaluation of dynamic quantities.

This example focuses on the validity of the approach using quadratic shape functions. A dynamic simulation with a load spatially moving with a frequency of $1000 \mathrm{~Hz}$ is performed and special attention is given to energy related quantities. Four different models have been used for comparison:

- FE: The unreduced mass and stiffness FE matrices are used. The total number of DOFs and equations of motion after application of the boundary conditions is 1520 , see eq.8. This simulation is used as reference for comparison with the other reduction techniques;

- 2 eig + 81 static: This reduced model includes the first 2 eigenmodes of the structure. These are included to provide an accurate dynamic description within the frequency range of interest considering that the external load motion is below the first resonance frequency. The model is augmented with residual attachment modes obtained by applying the load distribution depicted in fig. 3 with unit amplitude at 81 different discrete locations along the top edge of the rectangular block. Afterwards, the orthonormalization procedure in $[4,5]$ is followed. The 81 locations are chosen by placing the center of the load distribution on each 


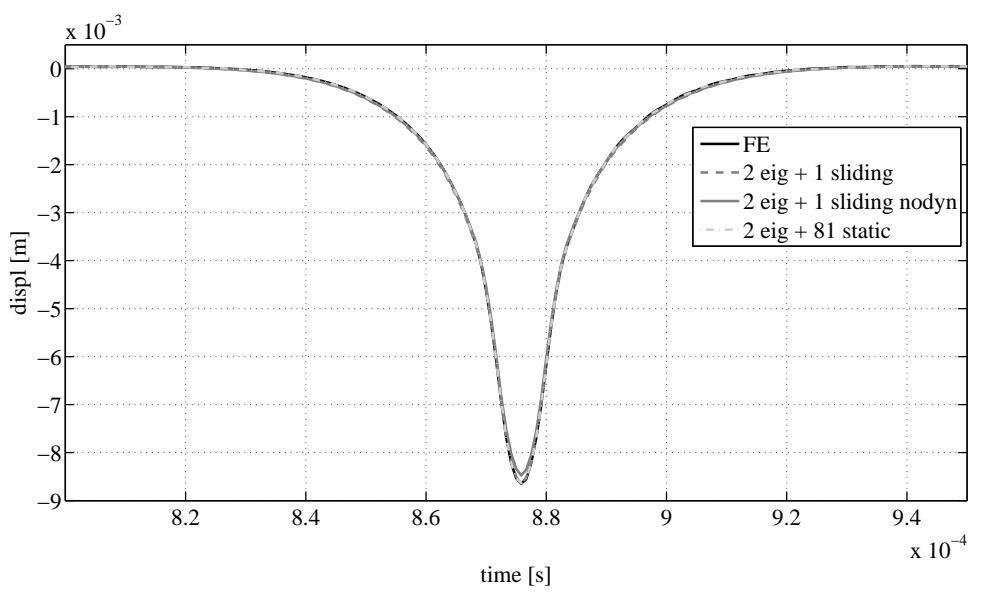

Figure 4: displacement at node $A_{1}$ in the $y$-direction

of the possible nodes that can be loaded during simulation. The 81 residual attachment modes differ from conventional ones simply by the fact that a load distribution is applied instead of a single point-load vector. This model shows that when highly dynamic loads are present, the inclusion of eigenmodes up to the frequency range of interest augmented with all the necessary residual attachment modes is a valid model reduction choice. This MOR scheme remains affordable only if the amount of external load locations remains limited. The total number of DOFs and equations of motion is 83 , see eq.2;

- 2 eig +1 sliding: This model is used to test the proposed PMOR scheme under dynamic conditions. It includes the above mentioned 2 eigenmodes and linear combinations of the 81 residual attachment modes according to eq.16. The final reduction space $\boldsymbol{V}$ contains a single parametric shape vector $\psi_{1}\left(p_{1}\right)$ (see eq.14) and 2 global shape vectors (the eigenmodes). The total number of DOFs and equations of motion is 3 , see eq.33;

- 2 eig +1 sliding nodyn: The reduced model is identical to the $\mathbf{2}$ eig $+\mathbf{1}$ sliding. The main difference lies in the fact that eq.34 is now solved by assuming $\dot{\boldsymbol{P}}$ and $\ddot{\boldsymbol{P}}$ equal to 0 . This corresponds to a matrix interpolation strategy that omits the extra terms obtained with the proposed formulation. This model is used to underline the importance of the extra terms in eq.33 in case of highly dynamic external loading.

Figure 4 shows the displacement at node $A_{1}$ in the $y$-direction in a time frame when the moving load crosses it. All the reduction schemes under analysis seem to give accurate deformation predictions, even at the load location. In particular the $\mathbf{2}$ eig $+\mathbf{1}$ sliding nodyn reduction does not seem to suffer the omission of the dynamic terms in eq.33 and shows a very accurate displacement evaluation. Only some minor deviations are noticeable when the displacement reaches its minimum. The investigation of dynamic quantities like local velocities highlights the importance of the extra terms in eq.33. In particular fig. 5 shows the $y$-direction velocity of node $B_{1}$. Despite the distance from the contact region, it is clear that the $\mathbf{2}$ eig $+\mathbf{1}$ sliding nodyn results are not accurate, especially around the time instants when the moving load crosses the central region of 


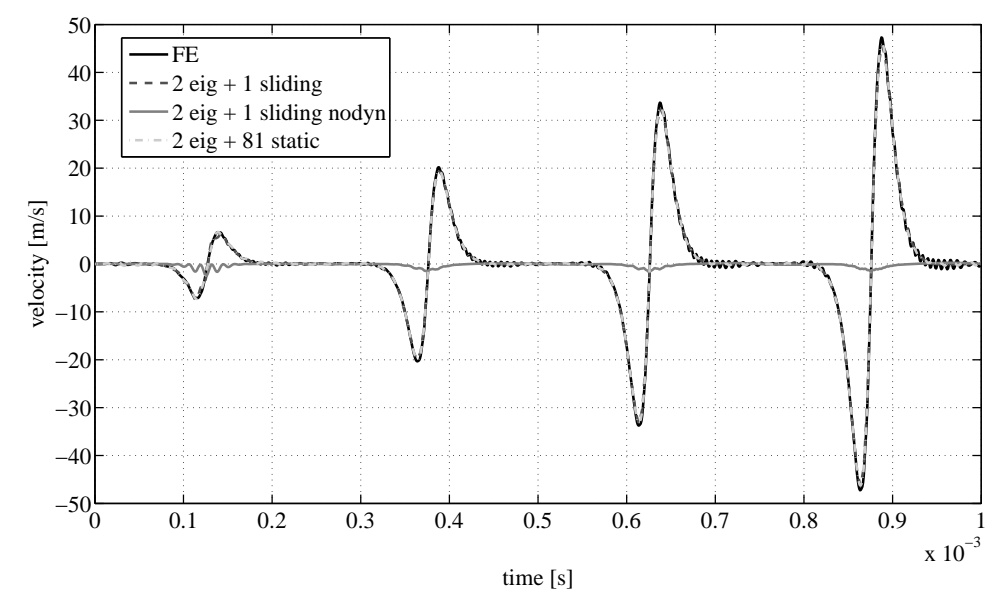

Figure 5: velocity at node $B_{1}$ in the $y$-direction
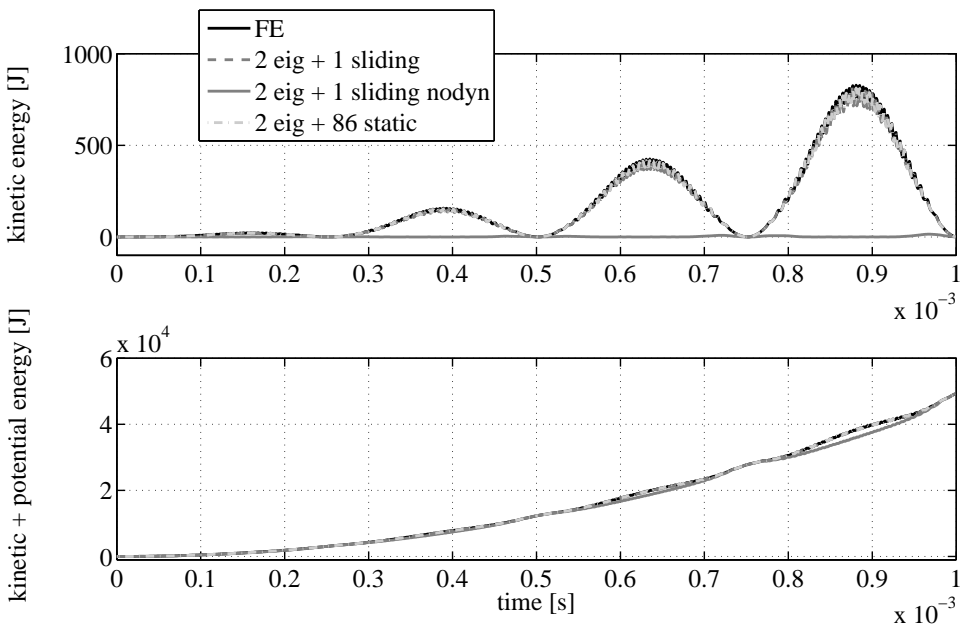

Figure 6: kinetic and total energy of the rectangular block

the rectangular block. The proposed $\mathbf{2}$ eig $+\mathbf{1}$ sliding time varying reduction is instead accurately matching the unreduced FE simulation and the $\mathbf{2}$ eig $+\mathbf{8 1}$ static results. In section 2.2 it was highlighted that the proposed technique should be energy preserving considering that it can be derived from an energetic principle. Given the fact that an integration scheme with dissipative properties has been used for time simulation, it is not possible to numerically support this statement. Nonetheless fig. 6 allows some considerations in that direction. The kinetic energy (top graph) that is instantaneously present in the system is largely underestimated by the $\mathbf{2}$ eig $+\mathbf{1}$ sliding nodyn reduction while the proposed time varying scheme shows a very similar behavior with respect to the reference models. By inspection of the sum of the kinetic and potential energy 
Table 3: Example $1 \mathrm{CPU}$ times

\begin{tabular}{|c|c|}
\hline Model used & CPU time $[s]$ \\
\hline \hline FE & 144.90 \\
\hline 2 eig + 81 static & 7.19 \\
\hline 2 eig + 1 sliding & 1.05 \\
\hline 2 eig + 1 sliding nodyn & 0.51 \\
\hline
\end{tabular}

in the system (bottom graph) it can be seen that the omission of the extra dynamic terms in eq.33 causes a different repartition between potential and kinetic energy, e.g. between $0.8 \cdot 10^{-3}$ and $0.9 \cdot 10^{-3} s$.

Table 3 quantifies the computational effort required for the simulations for each of the four different models. These values have been obtained by averaging 10 simulation repetitions for each case to improve consistency. The models are run in Matlab on a 64 bit machine equipped with two Intel(R) Xeon(R) X5605 $2.67 \mathrm{GHz}$ processors and $60 \mathrm{~GB}$ of RAM. Being aware of the implementation dependency, these values should be considered as qualitative, nonetheless a few observations can be drawn. The CPU time of the reference $\mathbf{F E}$ simulation is large compared to the reduced models (around $2 \mathrm{~min}$ and $20 \mathrm{~s}$ ). The proposed PMOR strategy on the contrary performs rather efficiently also with respect to the solution where all the attachment modes are included. More precisely the $\mathbf{2}$ eig $+\mathbf{1}$ sliding performs around 7 times faster than the $\mathbf{2}$ eig + 81 static and shows the same high simulation accuracy. Unsurprisingly the $\mathbf{2}$ eig $+\mathbf{1}$ sliding nodyn performs faster than the proposed $\mathbf{2}$ eig $+\mathbf{1}$ sliding technique. These results are even more favorable when a larger number of input points is present but less favorable when the parameters time histories are not a priori known.

To further reinforce this statement a different model, similar to the one presented in fig. 3 has been tested. The main difference lies in the width of the block that is extended with a factor 5 with respect to the original model. A similar load case has been applied to the the block surface. Given the larger width, the original FE mesh contains 3760 nodes (7520 DOF) and the number of possibly loaded nodes increased to 481. Four models have been evaluated: the full $\mathbf{F E}$ model (7520 DOF), a model reduced with 2 eigenmodes and 481 static modes (483 DOF) and two models for which the PMOR technique is applied. These models do not need extra residual attachment modes so that the number of DOF is equivalent to the $\mathbf{2}$ eig $+\mathbf{1}$ sliding and $\mathbf{2}$ eig +1 sliding nodyn cases for a total of 3 DOF each. The simulation time is extended to $0.012 s$. The accuracy of the results is comparable to the one already reported for the smaller block and is not presented for sake of brevity. Nonetheless the computational effort indicates the scalability of the performance when larger loaded interfaces are present. Table 4 shows that the CPU time of the unreduced model is prohibitively large $(2 h, 50 \mathrm{~m})$ and the model that includes all the attachment modes simulates in approximately $7 \mathrm{~min}$. The proposed PMOR strategy performs around 70 times faster than the latter while the model that does not include the extra dynamic terms performs 2 times faster than the model including these extra terms. Qualitatively these results are consistent with the ones reported in table 3 .

Summarizing, it can be stated that the proposed approach performs with good accuracy compared to the unreduced FE model. CPU time results show a significant time reduction. If the reduction space time-dependency is omitted in the equations of motion, results can be accurate at displacement level and computationally efficient. Nonetheless, dynamic quantities like nodal 
Table 4: Example 1 - longer block - CPU times

\begin{tabular}{|c|c|}
\hline Model used & CPU time $[s]$ \\
\hline \hline FE & 10223.1 \\
\hline 2 eig + 481 static & 419.4 \\
\hline 2 eig + 1 sliding & 6.3 \\
\hline 2 eig + 1 sliding nodyn & 3.4 \\
\hline
\end{tabular}

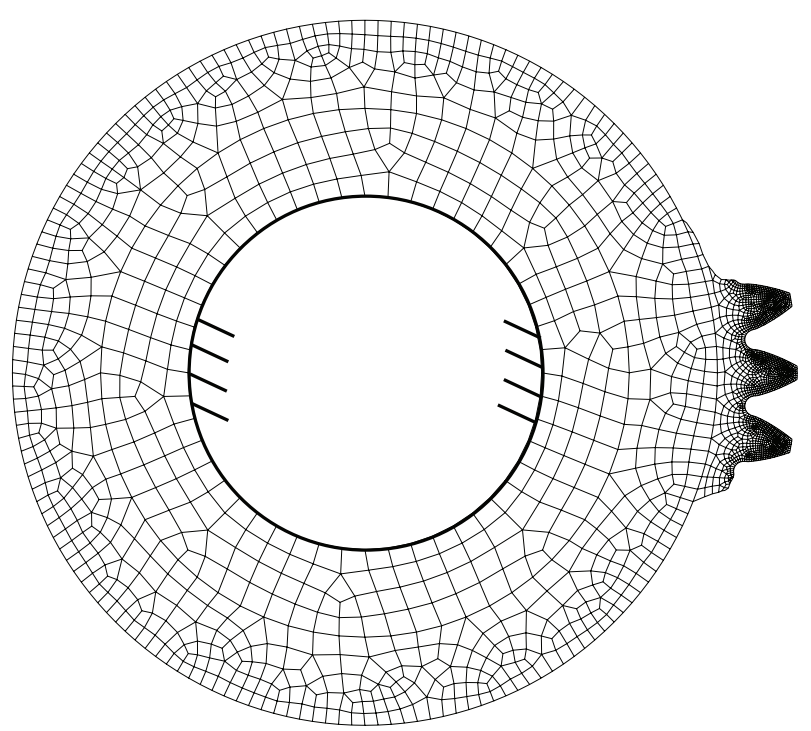

Figure 7: FE model

velocities can be wrongly assessed and energy repartition can be inconsistent for highly dynamic loading.

\subsection{Example 2: low frequency multiple point loads}

The second and third examples are performed on a 2 dimensional structural model of a spur gear. Figure 7 shows the gear mesh. The model is built using four noded membrane elements with bilinear shape functions. These elements are typically used for plane stress analysis. The gear center bore is clamped at all the nodes in both x and y DOFs. Different types of moving loads are then applied to one of the flanks of the central tooth. In order to reduce the large number of DOFs in the original gear, only 3 teeth are kept in the model. The reason for this is that the non-loaded teeth minimally affect the system response and, for the purpose of this study, mainly the effects related to the loaded tooth deformation are of relevance. The characteristics of the model are summarized in table 5. The time simulations use the same numerical scheme as in example 1 and the generalized- $\alpha$ damping parameter $\rho_{\infty}$ is chosen in order not to influence the simulation in the frequency range of interest. 
Table 5: general characteristics of the FE model used for simulation

\begin{tabular}{|c|c|}
\hline density $\left[\mathrm{kg} / \mathrm{m}^{3}\right]$ & 7860 \\
\hline weight $[\mathrm{kg}]$ & 1.234 \\
\hline thickness $[\mathrm{mm}]$ & 10 \\
\hline Young's modulus $[\mathrm{GPa}]$ & 210 \\
\hline Poisson's ratio & 0.3 \\
\hline total number of nodes & $2866(5732 \mathrm{DOF})$ \\
\hline number of clamped nodes & $48(96 \mathrm{DOF})$ \\
\hline $\begin{array}{c}\text { number of possibly loaded nodes } \\
\text { on tooth flank }\end{array}$ & $24(48 \mathrm{DOF})$ \\
\hline mode 1 frequency $[\mathrm{kHz}]$ & 11.61 \\
\hline mode 4 frequency $[\mathrm{kHz}]$ & 22.94 \\
\hline mode 200 frequency $[\mathrm{kHz}]$ & 149.44 \\
\hline
\end{tabular}

The main aim of this second example is to show the validity of the proposed PMOR approach when several external loads are applied to the systems. In this way the number of parameters is increased. Three point loads acting in the vertical direction are applied to the tooth flank, see fig.8. The loads act at different spatial frequencies and with different amplitudes as it can be seen from table 6. Since the highest spatial frequency of the moving loads is $1.13 \mathrm{~Hz}$ the time step is increased to keep the amount of integration points close to 10 when the load moves across each element edge, see table 6 . The choice of such a conservative time step is made in order to assure a smooth transition from one element to another avoiding abrupt discontinuities that may arise due to the use of linear shape functions in the FE model. The three loads $\boldsymbol{F}_{\boldsymbol{e x t}, \boldsymbol{i}}$ starts from an amplitude $F_{\text {amp }, i}=0 \mathrm{~N}$ at time $t_{0}=0 \mathrm{~s}$ and increases up to a value of $F_{\max , i}$ that is kept constant after an initial build up, as seen in fig.9. The $x$ location of the load is given by the value of $x_{l}$. Equation 49 shows that the load starts from a position $c$ that is approximately at the midspan of the tooth flank and moves sinusoidally on the tooth flank with frequency $f_{i}$ and amplitude $x_{m a g}$. The parameters vector $\boldsymbol{p}$ has size 3 and each parameter $p_{i}$ varies between 0 and 1 depending on the relative position of the load with respect to the nodes adjacent to it, see eq.50.

$$
\begin{gathered}
x_{l}=c+x_{\text {mag }} \sin \left(2 \pi f_{i} t\right) \\
p_{1}=\frac{x_{l}-x_{\text {node } j}}{x_{\text {node } j+1}-x_{\text {node } j}} \\
F_{\text {amp }}=\left\{\begin{array}{c}
\frac{-F_{\text {max }, i}}{2}\left(1-\cos \left(\frac{\pi t}{T_{s}}\right)\right) ; 0 \leq t<T_{s} ; \quad 0 T_{s} \\
F_{\text {max }} ; t \geq
\end{array}\right.
\end{gathered}
$$

Four different models have been used for comparison in this example:

- FE: The full mass and stiffness FE matrices are used. The total number of DOFs and equations of motion is 5636, see eq.8. This simulation is used as reference for comparison with the other reduction techniques;

- 200 eig: This reduced model is built by inclusion of the first 200 eigenmodes of the gear FE model computed with clamped boundary conditions. This model is used to show that the inclusion of global shape vectors up to very high-frequency with respect to the frequency 


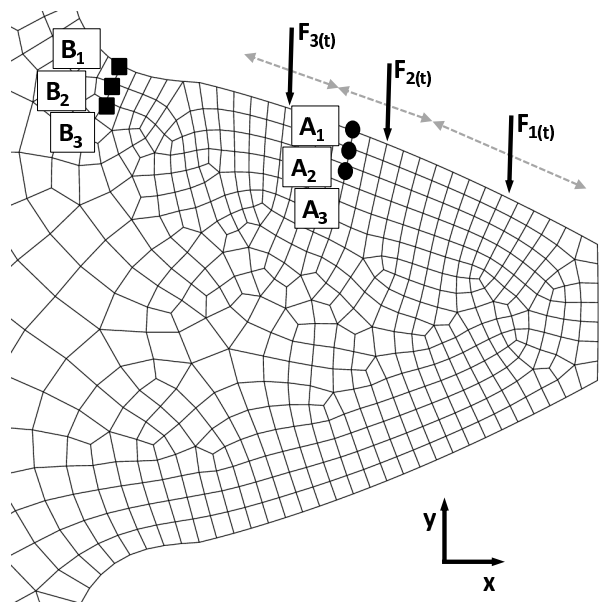

Figure 8: FE model, multiple moving load and virtual measurement locations

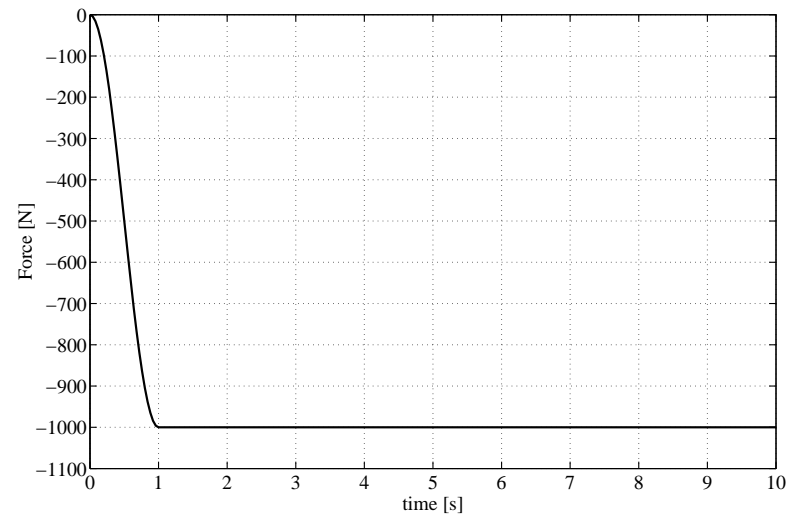

Figure 9: load amplitude $F_{\text {amp }}$

of interest might not be enough if local effects need to be considered. The total number of DOFs and equations of motion is 200 , see eq.2;

- 24 static: This reduced model is built by inclusion of all the attachment modes computed by applying unit loads on the 24 nodes of the tooth flank in the $y$-direction. No eigenmodes are included. This model shows that when quasi static conditions are present, the so called Guyan reduction [28] is a suitable solution that provides accurate results if all the attachment modes related to possible load locations are included. The total number of DOFs and equations of motion is 24 , see eq. 2 ;

- 3 sliding: This model is used to test the proposed PMOR scheme. It includes linear combinations of the above mentioned 24 attachment modes to create, according to eq.15, a reduction space $\boldsymbol{V}$. Since three external loads are presents the reduction space $\boldsymbol{V}$ contains 
Table 6: example 2 simulation details

\begin{tabular}{|c|c|}
\hline simulation time $[s]$ & 10 \\
\hline spectral radius at $\infty \rho_{\infty}$ & 0.5 \\
\hline time step $h[s]$ & $0.5 \cdot 10^{-2}$ \\
\hline $\begin{array}{c}\text { initial load positions } \\
c_{1}-c_{2}-c_{3}[\mathrm{~m}]\end{array}$ & $0.0955-0.0924-0.0899$ \\
\hline $\begin{array}{c}\text { load position amplitude } \\
x_{1 \mathrm{mag}}-x_{2} \text { mag }-x_{3} \operatorname{mag}[\mathrm{m}]\end{array}$ & $0.2 \cdot 10^{-2}-0.1 \cdot 10^{-2}-0.1 \cdot 10^{-2}$ \\
\hline $\begin{array}{c}\text { load position frequency } \\
f_{1}-f_{2}-f_{3}[H z]\end{array}$ & $0.1-0.53-1.13$ \\
\hline $\begin{array}{c}\text { load maximum amplitude } \\
F_{1 \max }-F_{2} \max \end{array}-F_{3 \max }[N]$ & $10^{3}-5 \cdot 10^{2}-5 \cdot 10^{2}$ \\
\hline Load raising time $T_{s}[s]$ & 1 \\
\hline
\end{tabular}

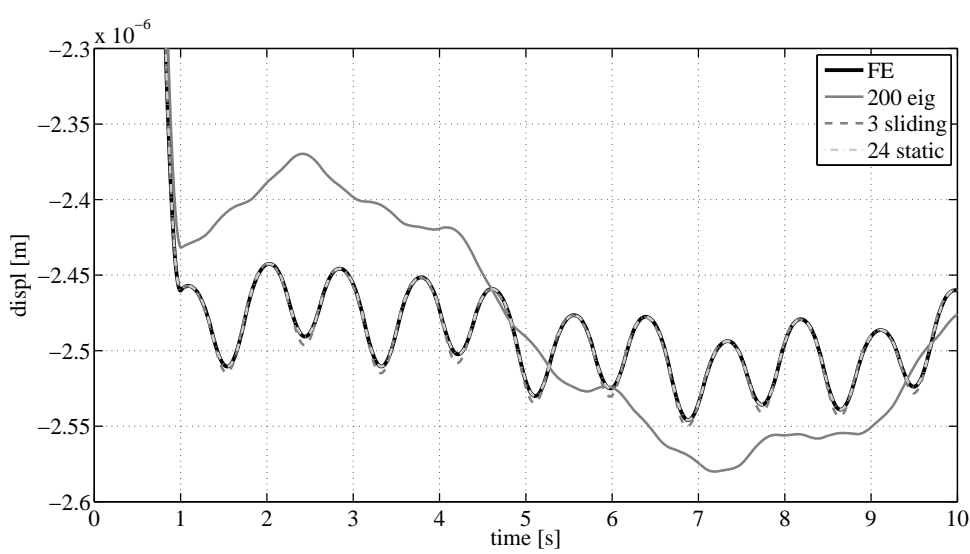

Figure 10: displacement at node $B_{1}$ in the tooth normal direction

three shape vectors $\left[\psi_{1}\left(p_{1}\right) \psi_{2}\left(p_{2}\right) \psi_{3}\left(p_{3}\right)\right]$ while no eigenmodes are included. The total number of DOFs and equations of motion is 3 , see eq. 33 .

The displacement response at the tooth root is depicted in fig.10. The $\mathbf{2 0 0}$ eig solution presents a low accuracy. Even when multiple parameters are present the proposed PMOR approach provides satisfactory results with only 3 DOFs and 3 scalar equations of motion. Frequently in gear applications, it is interesting to analyze the stresses at the contact location and tooth root. The time history of the Von Mises stress at the center of elements $A_{1}, A_{2}$ and $A_{3}$ is presented in fig.11. The local stresses experience peaks when the loads pass in the vicinity of the node $A_{1}$. The solution using only eigenmodes underestimates the peak stress considerably while the proposed PMOR approach is capable of accurately representing the local stresses with a degree of accuracy that is similar to the reference $\mathbf{F E}$ and $\mathbf{2 4}$ static models as expected. By further analyzing the results, it can be seen from fig. 12 that the stresses at the tooth root are also not correctly captured if no attachment modes are included in the simulation. Once more the proposed 


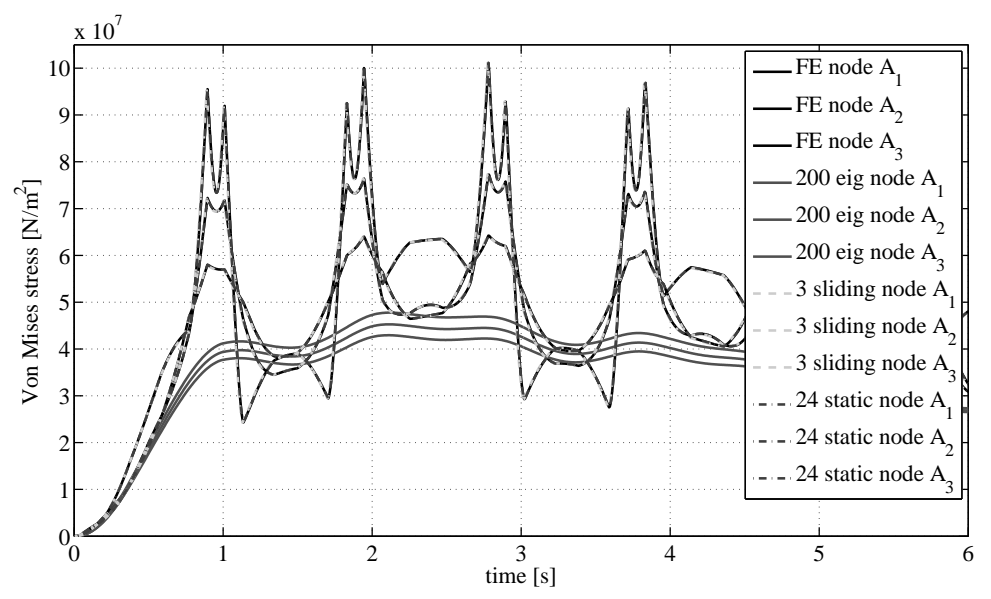

Figure 11: Von Mises stress at center of elements $A_{1}, A_{2}, A_{3}$

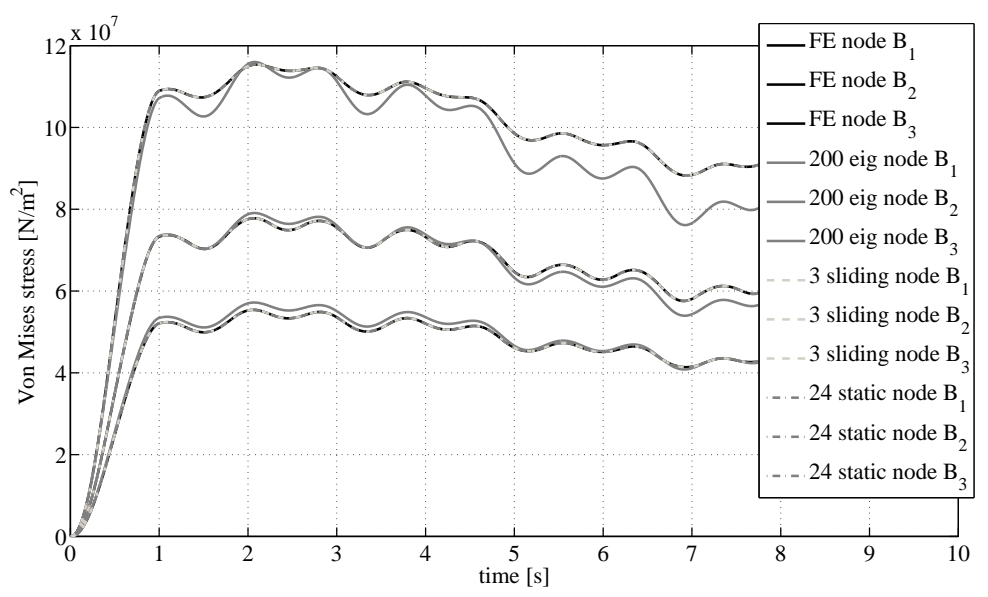

Figure 12: Von Mises stress at center of elements $B_{1}, B_{2}, B_{3}$

PMOR approach gives a satisfactory stress accuracy with respect to the FE model and $\mathbf{2 4}$ static.

Finally, to reinforce the previous statements, fig.13 a), c) and e) show a snapshot of the full Von Mises stress field after $4 s$ of simulation while fig. $13 \mathrm{~b}$ ), d) and f) show the Von mises stress field after $9 s$ of simulation for three different models. By visually comparing the results of the 200 eig against the reference simulation and the PMOR solution it is apparent how the complex stress concentration patterns around the load locations is not captured by using only eigenmodes while the proposed PMOR strategy closely reproduces the local stress field. The CPU times related to the simulations using each model are reported in table 7 . These values have been obtained by averaging 10 simulation repetitions for each case to improve consistency. The same equipment as for example 1 has been used. The CPU time of the reference FE simulation, as 

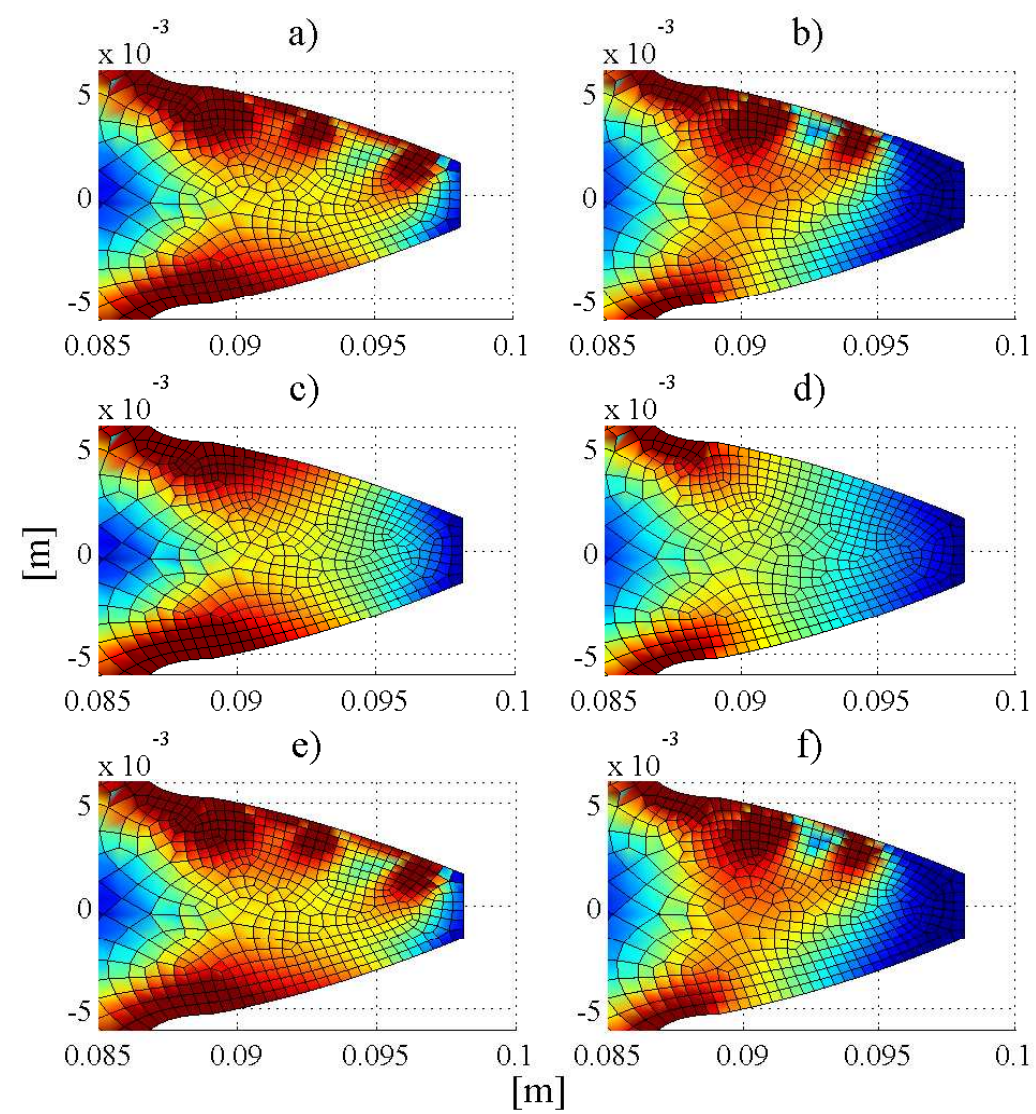

Figure 13: Von Mises stress field a) FE model after $4 s$ b) FE model after $9 s$ c) 200 eig model after $4 s$ d) 200 eig model after $9 s$ e) $\mathbf{3}$ sliding model after $4 s$ f) $\mathbf{3}$ sliding model after $9 s$

expected, large compared to the reduced models (around $20 \mathrm{~min}$ ) and the $\mathbf{2 0 0}$ eig is not able to perform in real time for this example. The proposed PMOR strategy on the contrary performs rather efficiently also with respect to the solution where all the attachment modes are included. More precisely the $\mathbf{3}$ sliding performs 18.5 times faster than real-time despite the very small time step chosen.

\subsection{Example 3: high frequency moving single load distribution}

A third example is used to show the behavior of the proposed PMOR when a highly dynamic, a priori known load distribution is applied to the tooth flank. The external load distribution $F_{\text {ext }}$ is depicted in fig. 14 where it can be seen that five point loads closely spaced with a fixed spacing, $f_{\text {ext }}^{i}$, are applied to the tooth flank to simulate a smooth load distribution. The load distribution is applied in a direction that form an angle of $68.48^{\circ}$ with the $x$ direction so that it is directed towards the averaged tooth flank normal direction. 
Table 7: Example 2 CPU times

\begin{tabular}{|c|c|}
\hline Model used & CPU time $[s]$ \\
\hline \hline FE & 1151.26 \\
\hline 200 eig & 20.29 \\
\hline 24 static & 1.43 \\
\hline 3 sliding & 0.54 \\
\hline
\end{tabular}

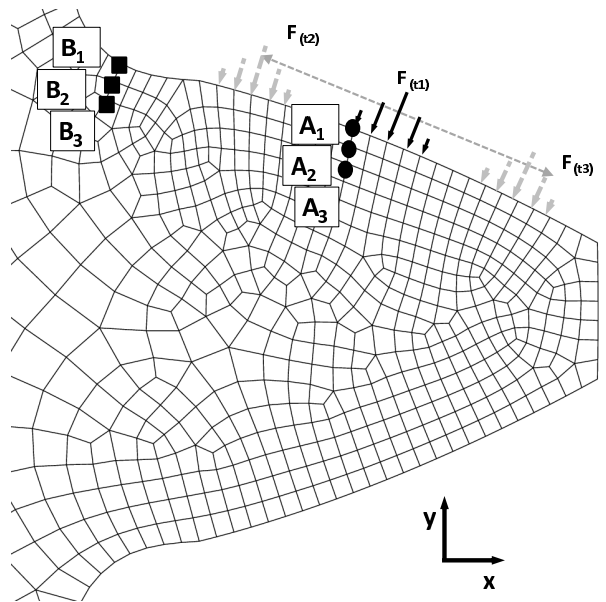

Figure 14: FE model, moving load distribution and virtual measurement locations

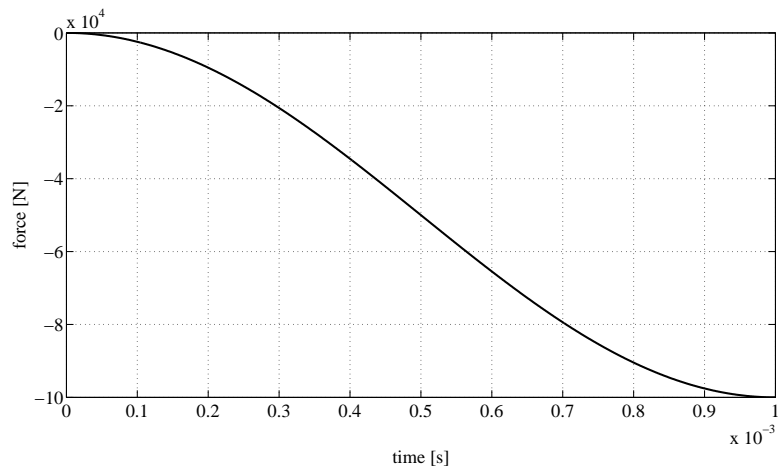

Figure 15: load amplitude $F_{\text {amp }}$

The amplitude $F_{\text {amp }}(t)$ is depicted in fig. 15 and defined by the parameters in table 8 . The 
Table 8: example 3 simulation details

\begin{tabular}{|c|c|}
\hline simulation time $[s]$ & $10^{-3}$ \\
\hline spectral radius at $\infty \rho_{\infty}$ & 0.5 \\
\hline time step $h[s]$ & $10^{-6}$ \\
\hline initial load positions $c[\mathrm{~m}]$ & 0.0935 \\
\hline load position amplitude $x_{\operatorname{mag}}[\mathrm{m}]$ & $0.15 \cdot 10^{-2}$ \\
\hline load position frequency $f_{1}[\mathrm{~Hz}]$ & $2 \cdot 10^{4}$ \\
\hline load maximum amplitude $F_{\max }[\mathrm{N}]$ & $10^{6}$ \\
\hline Load raising time $T_{s}[\mathrm{~s}]$ & $10^{-3}$ \\
\hline
\end{tabular}

amplitudes of the five point loads are related to this function by the following:

$$
\begin{aligned}
& f_{\text {ext }}^{1,5}(t)=F_{\text {amp }}(t) \sin \left(\frac{\pi}{18}\right), \\
& f_{\text {ext }}^{2,4}(t)=F_{\text {amp }}(t) \sin \left(\frac{5 \pi}{18}\right), \\
& f_{\text {ext }}^{3}(t)=F_{\text {amp }}(t) \sin \left(\frac{\pi}{2}\right) .
\end{aligned}
$$

Since the spatial frequency of the load distribution is increased to $20 \mathrm{kHz}$ the time step is reduced to maintain the amount of integration points close to 10 when the load moves along each element edge length. The frequency of $20 \mathrm{kHz}$ is chosen so that the system is loaded in its dynamic range between the eigenfrequencies of eigenmodes 3 and 4. For this example five different models have been used for comparison:

- FE: This model remains the same as in the previous examples;

- 200 eig: This model remains the same as in the previous examples. Given the fact that the load acts at $20 \mathrm{kHz}$ and that eigenmode 200 has an eigenfrequency of $149.44 \mathrm{kHz}$, this model still represents a very large reduction space if compared to the frequency range of interest that is approximately $0-40 \mathrm{kHz}$ ).

- 26 eig + 20 static: This reduced model includes the first 26 eigenmodes of the structure. This allows for an accurate description of the frequency range of interest given that mode 26 has an eigenfrequency of $50.01 \mathrm{kHz}$. The model is then augmented with residual attachment modes obtained by applying the load distribution depicted in fig. 14 with unit amplitude at 20 different discrete locations along the tooth flank and following the orthonormalization procedure in $[4,5]$. The 20 locations are chosen by placing the central force $f_{\text {ext }}^{3}$ on each of the possible nodes that can be loaded during simulation. Similarly to the $\mathbf{2}$ eig $+\mathbf{8 1}$ static in example 1, this model should be considered as a good reduction option if the amount of external load locations is limited. The total number of DOF and equations of motion is 46 , see eq. 2 ;

- 26 eig + 1 sliding: This model is used to test the proposed PMOR scheme under dynamic conditions. It includes the above mentioned 26 eigenmodes and linear combinations of the 20 residual attachment modes to create, according to eq.15, a reduction space $\boldsymbol{V}$ that 


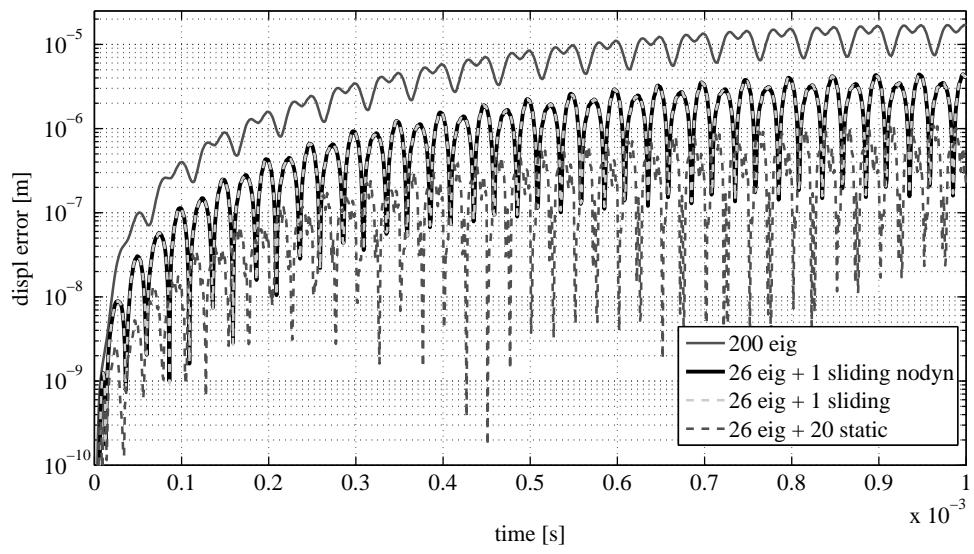

Figure 16: displacement error at node $B_{1}$ in the tooth normal direction

contains a single parametric shape vector $\psi_{1}\left(p_{1}\right)$ (see eq.14) together with some global shape vectors (the eigenmodes). The total number of DOF and equations of motion is 27 , see eq.33;

- 26 eig +1 sliding nodyn: The reduced model is identical to the 26 eig +1 sliding but eq.33 is now solved by assuming $\dot{\boldsymbol{P}}$ and $\ddot{\boldsymbol{P}}$ equal to 0 so that the effect related to the reduction space time-dependency are neglected.

Figure 16 shows the displacement error of the different models with respect to the reference $\mathbf{F E}$ simulation, evaluated at a root node in the tooth normal direction. It can be seen how the $\mathbf{2 0 0}$ eig model shows its lower quality with respect to the other three models. In particular by including all the residual attachment modes, the match with the reference simulation is high. The $\mathbf{2 6}$ eig + 1 sliding model behaves in a satisfactory way considering that it includes approximately half of the variables with respect to the $\mathbf{2 6}$ eig $+\mathbf{2 0}$ static simulation. Similar accuracy is expected also in the case in which the loading surface is wider, leading to more than just 20 residual attachment modes. Similarly to example 1 , the $\mathbf{2 6}$ eig $+\mathbf{1}$ sliding nodyn model behaves in a very similar way with respect to its dynamic PMOR counterpart if dynamic quantities are not inspected.

Figure 17 shows a representative zoom of the evolution of the system kinetic energy during the time simulation. The proposed PMOR strategy matches the system kinetic energy accurately while clearly the $\mathbf{2 6}$ eig $+\mathbf{1}$ sliding nodyn is incapable of performing with similar accuracy. These differences can be further highlighted by plotting the time history of the velocity of node $B_{1}$ in the tooth normal direction, see fig.18. It can be seen that the simpler matrix interpolation method, provides poor accuracy at velocity level while the $\mathbf{2 6}$ eig $+\mathbf{1}$ sliding reduction accurately captures the local dynamic behavior. It is felt that in many FE applications, where parameter variations can be expected to be small, the correct evaluation of displacement and stresses might not show significant benefits by adopting the proposed strategy. Nonetheless, in more complex non-linear systems like constrained multibody systems where the dynamics is highly coupled or in systems in which reaction loads, velocities and accelerations are of interest, the matrix interpolation results should be analyzed with care. Finally fig. 19 shows a representative zoom of the time histories of the Von Mises stresses evaluated at the center of elements $A_{1}, A_{2}$ and $A_{3}$. 


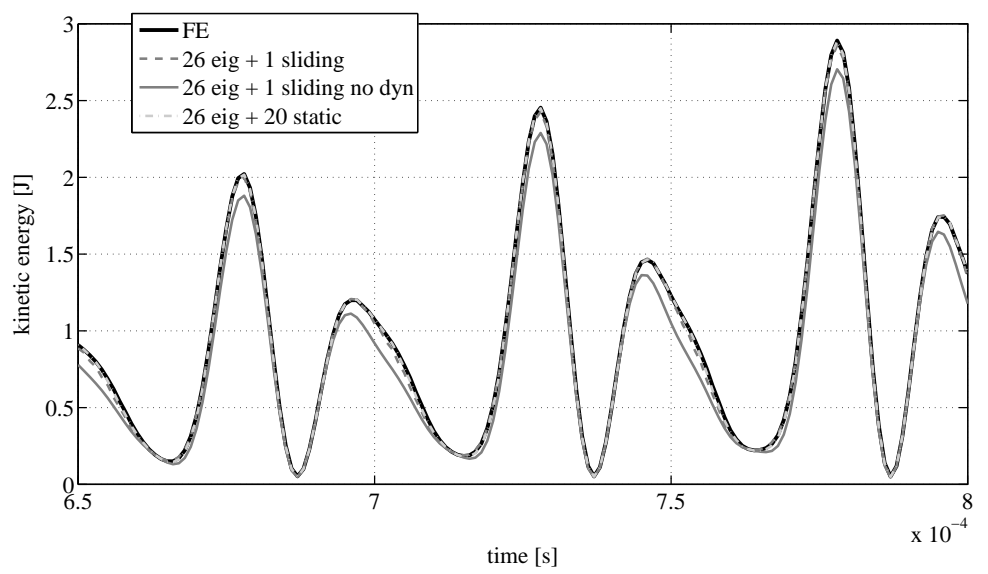

Figure 17: zoom of the system kinetic energy

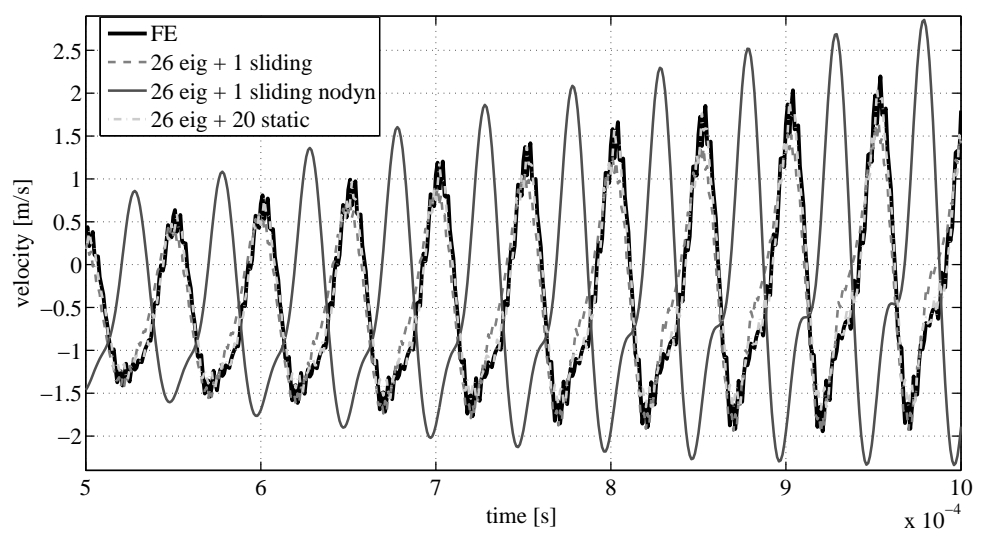

Figure 18: zoom of the velocity at node $B_{1}$ in the tooth normal direction

The proposed PMOR approach clearly captures local effects while the $\mathbf{2 0 0}$ eig solution fails to give the desired results.

\section{Conclusions}

This work introduces a novel time dependent parametric model order reduction scheme that can be used for simulation of systems with moving external loads and/or boundary conditions. Contrary to other approaches, the method does not rely only on reduced matrix interpolation but can be developed starting from an energetic principle. The obtained parametric equations of motion, see eq.34, show that some extra terms appear in order to take into consideration the dynamic change in reduction space that is performed on-line during simulation. In this way the formulation can be made efficient due to a consistent reduction in amount of degrees of freedom 


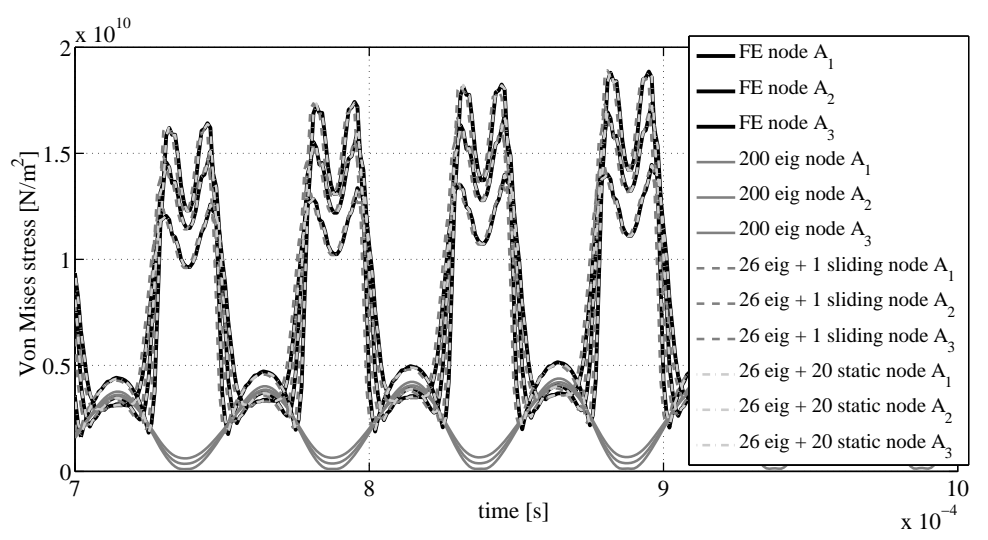

Figure 19: Von Mises stress at center of elements $A_{1}, A_{2}, A_{3}$

and by exploiting some desirable characteristics of the parametric shape vectors. The method is appealing for systems in which several external load locations are present but only few of them are active at a certain moment in time.

The method has been tested on three different numerical examples by using two different structural dynamic systems with linear and quadratic shape functions and several different moving external loads. Results show that the proposed PMOR strategy performs with the desired accuracy with respect to the reference FE models. In particular, example 1 highlights the advantages of the approach when dynamic quantities have to be evaluated and shows some interesting results regarding energy consistency and CPU time. Example 2 analyzes the behavior of the PMOR approach when multiple external moving loads and time varying shape vectors are present. The averaged CPU time evaluations show the benefits of the technique regarding efficiency leading to real-time performance for the proposed example. Example 3 is presented to show the capability of the method when a highly dynamic moving external load is applied to the system. Results related to dynamic stress evaluation are reported. The importance of the extra terms present in eq.34 are furthermore highlighted by comparing global kinetic energy and local velocities. Results of this investigation show that PMOR techniques by matrix interpolation provide satisfactory results both at displacement and stress level but present some limitations for the evaluation of dynamic quantities including velocities and kinetic energy.

Future work will concentrate on extending the proposed scheme to non-linear, highly coupled systems, with focus on cases in which the shape vectors are implicitly dependent on the reduced coordinates themselves. Applications to gear and bearing simulation are foreseen.

\section{ACKNOWLEDGEMENTS}

The IWT Flanders is gratefully acknowledged for its support within the O\&O MODRIO and SBO OptiWind projects. This work benefits also from the Belgian Programme on Interuniversity Attraction Poles, initiated by the Belgian Federal Science Policy Office (DYSCO). The research of Gert Heirman is funded by a OZM fellowship of the Institute for the Promotion of Innovation through Science and Technology in Flanders (IWT-Flanders). P. Ziegler and P. Eberhard 
of the Institute of Engineering and Computational Mechanics, University of Stuttgart, are also acknowledged for kindly providing the original gear mesh used for the numerical experiments.

\section{References}

[1] G. Heirman, W. Desmet, Interface reduction of flexible bodies for efficient modeling of body flexibility in multibody dynamics, Multibody System Dynamics 24 (2) (2010) 219-234.

[2] S. Donders, B. Pluymers, P. Ragnarsson, R. Hadjit, W. Desmet, The wave-based substructuring approach for the efficient description of interface dynamics in substructuring, Journal of Sound and Vibration 329 (8) (2010) 1062 1080.

[3] R. Craig, A review of time-domain and frequency-domain component mode synthesis methods, Journal of Modal Analysis 2 (2) (1987) 59-72.

[4] G. Heirman, T. Tamarozzi, W. Desmet, Static modes switching for more efficient flexible multibody simulation, International Journal for Numerical Methods in Engineering 87 (11) (2011) 1024-1045.

[5] T. Tamarozzi, P. Ziegler, P. Eberhard, W. Desmet, Static modes switching in gear contact simulation, Mechanism and Machine Theory Accepted for publication.

[6] T. Tamarozzi, G. Heirman, W. Desmet, Static modes switching: On-line variable static augmentation for efficient flexible multibody simulation, in: Proceedings of ASME IDETC/CIE 2011, Washington DC, 2011.

[7] K. Sherif, W. Witteveen, K. Mayrhofer, Quasi-static consideration of high-frequency modes for more efficient flexible multibody simulations, Acta Mechanica (2012) 1-21.

[8] A. Antoulas, Approximation of large-scale dynamical systems, Vol. 6, Society for Industrial Mathematics, 2005.

[9] J. Fehr, Automated Error Controlled Model Reduction in Elastic Multibody Systems, Dissertation, Schriften aus dem Institut für Technische und Numerische Mechanik der Universität Stuttgart, Volume 21, Shaker Verlag, Aachen, 2011.

[10] G. Kerschen, J. Golinval, A. Vakakis, L. Bergman, The method of proper orthogonal decomposition for dynamical characterization and order reduction of mechanical systems: an overview, Nonlinear Dynamics 41 (1) (2005) 147169.

[11] E. Balmès, Parametric families of reduced finite element models. theory and applications, Mechanical Systems and Signal Processing 10 (4) (1996) 381-394.

[12] J. Mohammadpour, K. Grigoriadis, Efficient modeling and control of large-scale systems, Springer, 2010.

[13] T. Bui-Thanh, K. Willcox, O. Ghattas, Model reduction for large-scale systems with high-dimensional parametric input space, SIAM Journal on Scientific Computing 30 (6) (2008) 3270-3288.

[14] H. Panzer, J. Mohring, R. Eid, B. Lohmann, Parametric model order reduction by matrix interpolation, atAutomatisierungstechnik 58 (8) (2010) 475-484.

[15] D. Amsallem, C. Farhat, An online method for interpolating linear parametric reduced-order models, SIAM Journal on Scientific Computing 33 (5) (2011) 2169-2198.

[16] U. Baur, C. Beattie, P. Benner, S. Gugercin, Interpolatory projection methods for parameterized model reduction, SIAM Journal on Scientific Computing 33 (5) (2011) 2489-2518.

[17] A. Leung, R. Khazaka, Parametric model order reduction technique for design optimization, in: Circuits and Systems, 2005. ISCAS 2005. IEEE International Symposium on, IEEE, 2005, pp. 1290-1293.

[18] M. J. Rewienski, A trajectory piecewise-linear approach to model order reduction of nonlinear dynamical systems, Ph.D. thesis, Citeseer (2003).

[19] M. Rewienski, J. White, A trajectory piecewise-linear approach to model order reduction and fast simulation of nonlinear circuits and micromachined devices, Computer-Aided Design of Integrated Circuits and Systems, IEEE Transactions on 22 (2) (2003) 155-170.

[20] O. Brüls, P. Duysinx, J. Golinval, The global modal parameterization for non-linear model-order reduction in flexible multibody dynamics, International journal for numerical methods in engineering 69 (5) (2007) 948-977.

[21] G. Heirman, F. Naets, W. Desmet, A system-level model reduction technique for the efficient simulation of flexible multibody systems, International Journal for Numerical Methods in Engineering 85 (3) (2011) 330-354.

[22] F. Naets, T. Tamarozzi, G. Heirman, W. Desmet, Real-time flexible multibody simulation with global modal parameterization, Multibody System Dynamics (2012) 1-18.

[23] R. Schwertassek, O. Wallrapp, A. Shabana, Flexible multibody simulation and choice of shape functions, Nonlinear Dynamics 20 (4) (1999) 361-380.

[24] R. Schwertassek, S. Dombrowski, O. Wallrapp, Modal representation of stress in flexible multibody simulation, Nonlinear Dynamics 20 (4) (1999) 381-399.

[25] C. Tobias, P. Eberhard, Stress recovery with krylov-subspaces in reduced elastic multibody systems, Multibody System Dynamics 25 (4) (2011) 377-393. 
[26] D. Amsallem, J. Cortial, K. Carlberg, C. Farhat, A method for interpolating on manifolds structural dynamics reduced-order models, International journal for numerical methods in engineering 80 (9) (2009) 1241-1258.

[27] M. Arnold, O. Bruls, Convergence of the generalized- $\alpha$ scheme for constrained mechanical systems, Multibody System Dynamics 18 (2) (2007) 185-202.

[28] R. Guyan, Reduction of stiffness and mass matrices(simultaneous stiffness and nondiagonal mass matrix reduction in structural analysis), AIAA Journal 3 (2) (1965) 380. 